THE ASTROPHYSICAL JOURNAL, 336:212-230, 1989 January 1

(C) 1989. The American Astronomical Society. All rights reserved. Printed in U.S.A.

\title{
HIGH-RESOLUTION OBSERVATIONS OF INTERSTELLAR Na I AND Ca II ABSORPTION LINES TOWARD THE SCORPIUS OB1 ASSOCIATION
}

\author{
I. A. Crawford and M. J. Barlow \\ Department of Physics and Astronomy, University College London \\ AND \\ J. C. BLADES \\ Space Telescope Science Institute \\ Received 1988 January 7; accepted 1988 June 17
}

\begin{abstract}
We present high-resolution observations $\left(R \geq 10^{5}\right)$ of interstellar $\mathrm{Na}$ I and $\mathrm{Ca}$ II toward 22 stars in and around the Sco OB1 association (13 association members, eight stars nearby on the sky, and one foreground star). The observed velocity structure, velocity dispersions, column densities, and $N(\mathrm{Na}$ I) $/ N(\mathrm{Ca}$ II) ratios are discussed. The velocity components observed toward each of the association members were found to be separable into three distinct groups: (1) strong absorptions (fully saturated in the case of $\mathrm{Na}$ I), extending from zero heliocentric velocity up to the association radial velocity of about $-20 \mathrm{~km} \mathrm{~s}^{-1} ;(2)$ discrete and generally sharp components with blueshifted velocities of up to about $-50 \mathrm{~km} \mathrm{~s}^{-1}$; (3) absorptions at positive heliocentric velocities (extending up to about $+20 \mathrm{~km} \mathrm{~s}^{-1}$ ). The $N(\mathrm{Na} \mathrm{I}) / N(\mathrm{Ca}$ II) ratios were found to be different for these three velocity groups. The low negative velocity components have $\mathrm{Na}$ I/Ca II ratios ranging from 4 to 200 , consistent with the values found in the past for diffuse interstellar clouds, and so we interpret these components as due to diffuse clouds intercepted by the lines of sight to the Sco OB1 stars. The highly blueshifted material has much lower $\mathrm{Na}$ I/Ca II ratios, typically unity, which we interpret as being due to the partial removal of trapped calcium atoms from the surfaces of grains in a swept-up shell of material expanding away from the association. The positive velocity components have even lower $\mathrm{Na}$ I/Ca II ratios $(\leq 0.4)$. We interpret these components as arising from warm intercloud material and propose that such material can have velocities of up to $\pm 20 \mathrm{~km} \mathrm{~s}^{-1}$ relative to the local rest velocity along a line of sight. Positive velocity components due to this material are observable, whereas any velocity components between 0 and $-20 \mathrm{~km} \mathrm{~s}$ due to similar material are masked by the much stronger absorptions due to interstellar clouds. A dip seen in the $\mathrm{Na}$ I/Ca II ratios between -20 and $-40 \mathrm{~km} \mathrm{~s}^{-1}$ can, however, be interpreted as due to a blending between intercloud components and Sco OB1 shell components.
\end{abstract}

Subject headings: clusters: associations — interstellar: abundances — interstellar: matter

\section{INTRODUCTION}

Munch (1957) first drew attention to the unusually high negative velocities present in the interstellar gas seen toward OB associations, and this is now recognized to be a common phenomenon; see, for example, Walborn and Hesser (1975; Car OB1), Cowie, Songaila, and York (1979; Ori OB1), Phillips and Gondhalekar (1981; Per OB1), Phillips, Welsh, and Pettini (1984; Cyg OB1), and Whiteoak and Gardner (1985; Ara OB1).

The Sco OB1 association $\left(l=343^{\circ}, b=+1^{\circ}\right)$ is situated in the Sagittarius spiral arm at a distance of about $1900 \mathrm{pc}$ (Humphreys 1978). It is spread over a region of approximately $1^{\circ}$ by $2^{\circ}$, with the greatest elongation approximately in the Galactic plane, and contains the bright and compact open cluster NGC 6231, somewhat south of center. Photographs of the region can be found in Bok, Bok and Graham (1966). The association is very young, with an evolutionary age of about $(3.6 \pm 0.6) \times 10^{6}$ yr (van Genderen, Bijeveld, and van Groningen 1984) and, along with Car OB1 and Cyg OB2, contains one of the greatest concentrations of massive luminous stars in the Galaxy.

We report here observations of the interstellar $\mathrm{Na}$ I and $\mathrm{Ca}$ II lines toward a number of stars in and around this association. The relative positions of the stars observed are shown in Figure 1. The aim of this work was to compare the interstellar spectra observed towards stars within the association, in the foreground of the association, and nearby on the sky to the association. The velocity structure data, and the derived $N(\mathrm{Na}$ I $) / N(\mathrm{Ca}$ II) ratios, allowed us to distinguish three physical components: two in the general interstellar medium and one local to the association.

Unless otherwise stated, all velocities referred to in this paper are heliocentric.

\section{OBSERVATIONS}

All observations were made with the coudé echelle spectrograph of the Mount Stromlo Observatory $1.9 \mathrm{~m}$ telescope operating with the 130 inch $(3.3 \mathrm{~m})$ camera. High-resolution observations of the interstellar Na I D2 (5889.950 $\AA)$ line toward 11 stars in the Sco OB1 association, and another eight stars that are close to it on the sky, were obtained in $1978 \mathrm{July}$ and 1979 June. Observations of the interstellar Ca II K (3933.663 $\AA)$ line toward nine stars in the association were obtained in 1986 June, together with Na I D2 observations toward two additional stars. Details of the stars observed are given in Table 1 . In addition, a small number of lightly reddened stars (e.g. $\propto$ Vir, $E(B-V)=0.03 ; \zeta$ Pup, $E(B-V)=0.04, \mu$ Cen, $E(B-V)=0.08 ;$ and $\lambda$ Sco, $E(B-V)=0.03)$ were observed so as to have at least two spectra of the telluric absorption lines in this spectral region on 

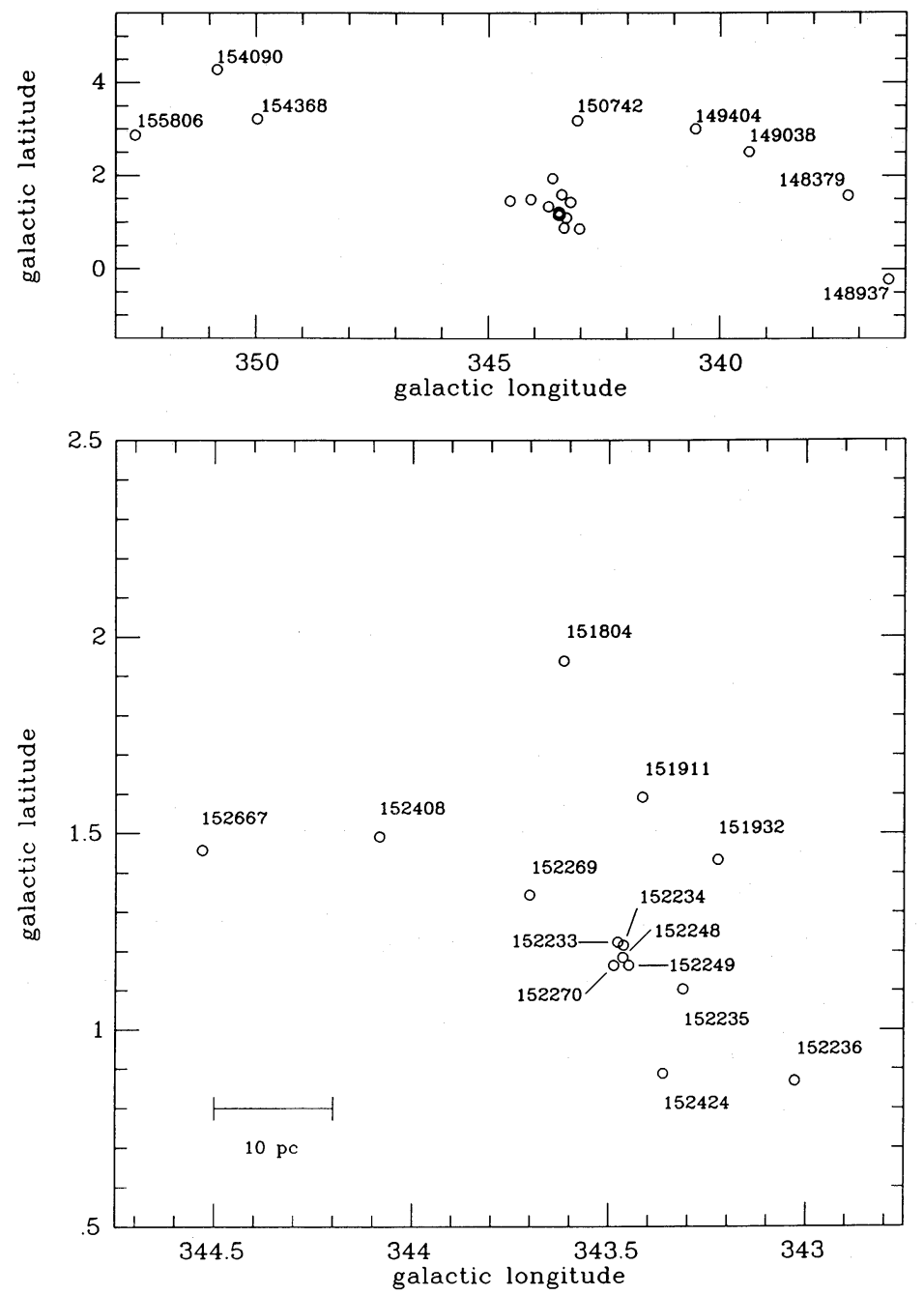

FIG. 1.-(top) Plot showing the relative positions of stars observed in this paper. The tight grouping of stars toward the center is the Sco OB1 association, the other stars (labeled with their HD numbers) are close to it on the sky but lie at a variety of distances (Table 1). (bottom) An expanded view of the Sco OB1 association members observed. The tight grouping of stars toward the center is the cluster NGC 6231. HD 152269 is a foreground star (see text). The scale bar is based on an assumed distance of $1900 \mathrm{pc}$

each night, particularly those of water vapor which are troublesome for high-resolution studies of $\mathrm{Na} \mathrm{D}$ (see Fig. 1 of Hobbs 1978a).

The 130 inch camera gives a dispersion at $\mathrm{Na}$ I D of $0.53 \AA$ $\mathrm{mm}^{-1}$ and an image scale of $5^{\prime \prime} 69 \mathrm{~mm}^{-1}$. The observations of 1978-1979 were made with a variety of slit widths in the range 125-250 $\mu \mathrm{m}$, giving velocity resolutions (FWHM of the instrumental response function, IRF) between 1.5 and $3.0 \mathrm{~km} \mathrm{~s}^{-1}$ (see Crawford, Rees, and Diego 1987). Details of the exposures made are given in Table 2. The detector used was an early (onedimensional) version of the Photon Counting Array (PCA) developed at Mount Stromlo (Stapinski, Rodgers, and Ellis 1979). The spectral range of the detector at $\mathrm{Na} D$ was about 6 $\AA$, and no lines from the then available comparison lamps were found in this range. Wavelength calibration was therefore performed by fitting a third-order polynomial to the positions of atmospheric water lines observed in the spectra of the unreddened stars. Five lines were identified and their wavelengths were taken from Moore, Minnaert, and Houtgast (1966). The wavelengths were then converted to heliocentric velocities. A guide to the accuracy of this procedure was obtained by com- paring the wavelength-calibrated spectra obtained of the same star in different years after performing the heliocentric velocity correction. This was possible in two cases, since HD 152234 and HD 152235 were observed in both 1978 and 1979 (although only the higher signal-to-noise ratio and/or higher resolution spectra were used for the final analysis). In both cases a small (1.38 and $1.26 \mathrm{~km} \mathrm{~s}^{-1}$, respectively) discrepancy was found, in the sense that the 1979 velocities are more negative than the 1978 velocities. We therefore estimate that this wavelength calibration is good to about $40 \mathrm{~m} \AA$ or $2 \mathrm{~km} \mathrm{~s}^{-1}$.

The format of the detector did not allow simultaneous sky monitoring and the zero intensity level was taken to be defined by the flat cores of the heavily saturated lines. Each spectrum was divided by a mean water vapor spectrum obtained by merging several of the unreddened stellar spectra. Little variation was observed from night to night in the atmospheric spectra, and it was considered best to merge several so as to improve the signal-to-noise ratio. In any case this telluric correction was found to have a negligible effect on the profiles of the sodium lines.

Inspection of the 1978-1979 $\mathrm{Na}$ I results revealed very 
TABLE 1

BASIC DATA FOR THE OBSERVED STARS

\begin{tabular}{|c|c|c|c|c|c|c|c|}
\hline $\begin{array}{c}\text { Star } \\
\text { (HD) }\end{array}$ & R.A.(1950.0) & Decl.(1950.0) & $V$ & $\mathrm{Sp}^{\mathrm{a}}$ & $E(B-V)^{\mathrm{b}}$ & $\begin{array}{l}\text { Distance }^{c} \\
\text { (pc) }\end{array}$ & Status \\
\hline 148379. & $16^{\mathrm{h}} 26^{\mathrm{m}} 04^{\mathrm{s}}$ & $-46^{\circ} 08^{\prime} 03^{\prime \prime}$ & 5.3 & B1.5 Iape & 0.76 & 1380 & Ara OB1a \\
\hline $148937 \ldots$ & 163010 & -480024 & 6.7 & O6 If & 0.67 & 1380 & Ara OB1a \\
\hline $149038 \ldots$ & 163031 & -435629 & 4.9 & B0 Ia & 0.31 & 1380 & Ara OB1a? \\
\hline $149404 \ldots \ldots \ldots \ldots$ & 163251 & -424527 & 5.5 & O9 Iae & 0.67 & 1380 & Ara OB1a \\
\hline $150742 \ldots$ & 164114 & -404451 & 5.7 & B3 V & 0.10 & 260 & field star \\
\hline $151804 \ldots$ & 164804 & -410848 & 5.3 & O8 Ifp & 0.35 & 1900 & Sco OB1 \\
\hline $151911 \ldots \ldots \ldots \ldots$ & 164848 & -413119 & 9.5 & B2 V & 0.40 & 1900 & Sco OB1 \\
\hline $151932 \ldots \ldots \ldots \ldots$ & 164848 & -414616 & 6.5 & WN7a & 0.45 & 1900 & Sco OB1 \\
\hline $152233 \ldots$ & 165032 & -414234 & 6.7 & O6 IIIfp & 0.41 & 1900 & NGC 6231 \\
\hline $152234 \ldots \ldots \ldots \ldots$ & 165031 & -414331 & 5.5 & B0.5 Ia & 0.46 & 1900 & NGC 6231 \\
\hline $152235 \ldots \ldots \ldots \ldots$ & 165028 & -415448 & 6.4 & B1 Iae & 0.79 & 1900 & NGC 6231 \\
\hline $152236 \ldots \ldots \ldots \ldots$ & 165028 & -421651 & 4.8 & B1 Iape & 0.69 & 1900 & Sco OB1 \\
\hline $152248 \ldots \ldots \ldots \ldots$ & 165039 & -414439 & 6.1 & O7fe & 0.44 & 1900 & NGC 6231 \\
\hline $152249 \ldots \ldots \ldots \ldots$ & 165041 & -414607 & 6.5 & O9 Ib & 0.48 & 1900 & NGC 6231 \\
\hline $152269 \ldots \ldots \ldots \ldots$ & 165048 & -412733 & 8.4 & B7 III & 0.26 & 720 & foreground \\
\hline $152270 \ldots \ldots \ldots \ldots$ & 165049 & -414421 & 6.7 & $\mathrm{WC7}+\mathrm{O} 5$ & 0.48 & 1900 & NGC 6231 \\
\hline $152408 \ldots \ldots \ldots \ldots$ & 165129 & -410415 & 5.8 & O8 If & 0.47 & 1900 & Sco OB1 \\
\hline $152424 \ldots \ldots \ldots \ldots$ & 165132 & -420039 & 6.4 & O9 Ia & 0.69 & 1900 & Sco OB1 \\
\hline $152667 \ldots \ldots \ldots \ldots$ & 165307 & -404443 & 6.2 & B0.5 Iae & 0.56 & 1900 & Sco OB1 \\
\hline $154090 \ldots \ldots \ldots \ldots$ & 170132 & -340316 & 4.9 & B1 Iae & 0.46 & 1040 & field star \\
\hline $154368 \ldots$ & 170308 & -352305 & 6.1 & O9.5 Iab & 0.78 & 860 & Sco OB4 \\
\hline $155806 \ldots \ldots \ldots \ldots$ & 171202 & -332933 & 5.5 & O8 IIIe & 0.33 & 900 & Sco OB4 \\
\hline
\end{tabular}

a Spectral types are from the Bright Star Catalogue (BSC; Hoffleit and Jaschek 1982) except for HDs 148937, 154368, and 155806, which are from Garrison et al. 1977.

${ }^{b}$ For the Sco OB1 members, the reddenings are from van Genderen et al. 1984. For the nonmembers they have been calculated from the spectral types and the photometry of either Schild et al. 1983 (HDs 148379, 148937, 154368, and 155806) or the BSC (HD 149038 and HD 150742), together with the intrinsic colors of Deutschman et al. 1986

c A distance of $1.9 \pm 0.3 \mathrm{kpc}$ has been adopted for the Sco OB1 association, including the central cluster NGC 6231 (Humphreys 1978). The distance to Ara OB1 is taken to be $1.38 \pm 0.2 \mathrm{kpc}$ (Humphreys 1978). HD 148379 and HD 148937 are probable members of Ara OB1 and HD 149038 a possible member, but see $\S$ IVe for contrary evidence. The adopted distance for HD 152269 comes from Schild et al. (1971). For the remaining stars the distances have been calculated using the absolute magnitude calibration of Deutschman et al. 1976 and $R=3.1$.

TABLE 2

Details of Exposures Made in the Na D2 Region toward MEMBERS OF SCO OB1 AND SurRounding Stars

\begin{tabular}{|c|c|c|c|}
\hline $\begin{array}{c}\text { Star } \\
\text { (HD) }\end{array}$ & $\begin{array}{c}\text { Exposure }^{a} \\
\text { (s) }\end{array}$ & $\begin{array}{c}\text { Slit } \\
(\mu \mathrm{m})\end{array}$ & $\begin{array}{c}\Delta v^{\mathrm{b}} \\
\left(\mathrm{km} \mathrm{s}^{-1}\right)\end{array}$ \\
\hline 148379 . & 3300 & 125 & 1.5 \\
\hline 148937. & 2000 & 250 & 3.0 \\
\hline 149038 & 1290 & 125 & 1.5 \\
\hline $149404 \ldots$ & 1200 & 250 & 3.0 \\
\hline $150742 \ldots$ & 2000 & 350 & 3.0 \\
\hline n........... & 5700 & 125 & 1.5 \\
\hline $151911 \ldots$ & 9580 & 250 & 3.0 \\
\hline $151932 \ldots$ & 3000 & 250 & 3.0 \\
\hline $152233 \ldots$ & 3000 & 250 & 3.0 \\
\hline 152234. & 2000 & 150 & 1.8 \\
\hline 152235 . & 3000 & 250 & 3.0 \\
\hline $152236 \ldots \ldots \ldots \ldots \ldots$ & 3000 & 125 & 1.5 \\
\hline $152249 \ldots \ldots \ldots \ldots \ldots$ & 3000 & 250 & 3.0 \\
\hline 152269 . & 9000 & 250 & 3.0 \\
\hline $152270 \ldots$ & 3000 & 250 & 3.0 \\
\hline$\ldots \ldots \ldots \ldots$ & 2000 & 150 & 1.8 \\
\hline$\ldots \ldots \ldots \ldots$ & 3000 & 250 & 3.0 \\
\hline $152667 \ldots$ & 2000 & 200 & 2.4 \\
\hline 154090. & 2000 & 125 & 1.5 \\
\hline $154368 \ldots \ldots \ldots \ldots \ldots \ldots$ & 2000 & 250 & 3.0 \\
\hline $155806 \ldots \ldots \ldots \ldots \ldots \ldots \ldots$ & 2000 & 200 & 2.4 \\
\hline
\end{tabular}

a Exposure times are for single exposures except for HD 151804 , where two have been merged.

${ }^{b} \Delta v$ is the FWHM of the instrumental response function in velocity units. It is calculated by convolving the grating interference pattern with the projected slit width (Crawford et al. 1987). complex velocity structures toward stars belonging to the association. In an attempt to determine how much of this structure is local to the association, the detailed photometry of van Genderen, Bijeveld, and van Groningen (1984) was examined for stars in the direction of Sco OB1 which they considered to be in the foreground. Two candidates, HD 151911 and HD 152269, were identified as being foreground and were observed in 1986 June. The resulting interstellar spectra (see below) indicated that HD 151911 is, after all, probably a member of the association, a conclusion supported by the stellar abundance analysis of Keenan et al. (1984). However, its interstellar spectrum and the work of Keenan et al. support the view that HD 152269 is a low-mass foreground star. A slit width of $250 \mu \mathrm{m}$ was used for these Na I D2 observations, and the exposure times were $9580 \mathrm{~s}$ (shared between two separate exposures) for HD 151911 and $9000 \mathrm{~s}$ for HD 152269. The detector was a more recent, two-dimensional, version of the PCA (Stapinski, Rodgers, and Ellis 1981) and a new thoriumargon lamp was available for wavelength calibration. Rest wavelengths were taken from Norlén (1973) and Giacchetti, Stanley, and Zalubas (1970). Two lines from this lamp were found to lie in the $6 \AA$ width of the detector and it was therefore necessary to linearly interpolate between them. Calculations based on the theoretical dispersion of the instrument indicate that the maximum error introduced by this approximation will amount to $\pm 5 \mathrm{~m} \AA$ or $0.26 \mathrm{~km} \mathrm{~s}^{-1}$, although this does not allow for any distortions due to the detector itself.

The two-dimensional PCA did permit the measuring of the background on either side of the spectrum. Following subtrac- 
tion of the background the saturated cores of the sodium lines were found to lie about $4 \%$ above the zero level. We attribute this to light scattered by the echelle and subtracted it also. The spectra were then divided by a flatfield and by an atmospheric water spectrum.

The instrumental setup used for the 1986 June Ca II observations was exactly the same as described above for the 1986 sodium observations. At the wavelength of the $\mathrm{Ca}$ II $\mathrm{K}$ line $(3933.663 \AA)$ the dispersion was $0.35 \AA \mathrm{mm}^{-1}$, and a $250 \mu \mathrm{m}$ slit again gave a velocity resolution of $3.0 \mathrm{~km} \mathrm{~s}^{-1}$. At this wavelength the detector had a spectral range of $4 \AA$, and once again only two lines from the Th-Ar lamp were found within it. The maximum error introduced by assuming a linear dispersion from these lines is again about $\pm 0.26 \mathrm{~km} \mathrm{~s}^{-1}$.

The background on either side was subtracted from each spectrum and the result divided by a flat field. Inspection of the spectra (Figs. 2 and 4 ) shows that the calcium lines are not fully saturated, and it is therefore necessary to trust that the subtraction of the background has given the proper zero level. The results for sodium discussed above indicate that this may be in error by about $4 \%$. The effect of this on the measurements is discussed in $\S$ III. Details of the exposures are given in Table 3.

\section{THE DERIVED Na I AND Ca II COLUMN DENSITIES}

\section{a) Line Profile Analysis}

In our analysis each cloud component was characterized by a radial velocity, $v$, column density of absorbers, $N$, and velocity dispersion parameter, $b$. The theory governing the line profiles as a function of $N$ and $b$ has been given by Strömgren (1948). We have used the interactive computer program BACH, developed originally by Davenhall (1977), on the UCL STARLINK node. This program makes use of Strömgren's analysis to calculate theoretical line profiles for a given $b$ and $N$. The profiles due to neighboring velocity components (in general with different values of $b$ and $N$ ) are blended together to model the whole absorption feature.

The $\mathrm{Na}$ I $\mathrm{D}$ lines are subject to hyperfine splitting (e.g., Wayte, Wynne-Jones, and Blades 1978, Blades, Wynne-Jones, and Wayte 1980), and this must be allowed for in the analysis. For the D2 line the splitting amounts to $19 \mathrm{~m} \AA$, or $0.97 \mathrm{~km}$ $\mathrm{s}^{-1}$. This is just below the resolution employed here, but it was nevertheless found to broaden the profiles. It was accounted for by performing the line profile calculation for each hyperfine component separately and then blending them together. The oscillator strength for the transition was taken to be 0.650 and

TABLE 3

Details of EXPosures MADE for INTERSTEllar Ca II toward Nine Stars in the SCo OB1 Association

\begin{tabular}{ccc}
\hline \hline $\begin{array}{c}\text { Star } \\
\text { (HD) }\end{array}$ & $\begin{array}{c}\text { Number of } \\
\text { Exposures }\end{array}$ & $\begin{array}{c}\text { Total Exposure } \\
\text { Time } \\
\text { (s) }\end{array}$ \\
\hline $151804 \ldots \ldots \ldots \ldots$ & 1 & 2500 \\
$152234 \ldots \ldots \ldots \ldots$. & 2 & 2500 \\
$152235 \ldots \ldots \ldots \ldots$. & 2 & 8300 \\
$152236 \ldots \ldots \ldots \ldots$. & 2 & 2000 \\
$152248 \ldots \ldots \ldots \ldots$. & 1 & 1500 \\
$152249 \ldots \ldots \ldots \ldots$. & 1 & 2500 \\
$152270 \ldots \ldots \ldots \ldots$. & 1 & 3500 \\
$152408 \ldots \ldots \ldots \ldots$. & 1 & 5400 \\
$152424 \ldots \ldots \ldots \ldots$. & 1 & 3600 \\
\hline
\end{tabular}

Note- -A slit width of $250 \mu \mathrm{m}$ was used for all exposures giving a velocity resolution of $3.0 \mathrm{~km} \mathrm{~s}^{-1}$. was scaled by the ratios of the statistical weights to get the relative strengths of the hyperfine components (Blades, Wynne-Jones, and Wayte 1980). The oscillator strength for the Ca II K line was taken to be 0.688 (Morton and Smith 1973). There is no hyperfine structure in calcium.

The calculated profiles were convolved with the IRF to enable comparison with the observations. The IRF of the Mount Stromlo coudé spectrograph has been discussed by Crawford, Rees, and Diego (1987) who concluded that the actual IRF is closely approximated by the ideal one calculated by convolving the grating interference pattern by the projected slit width. This is the IRF used in the subsequent modelling.

The parameters $v, b$, and $N$ were adjusted by trial and error until a satisfactory fit to each spectrum was obtained. The best fits for each star are shown in Figures 2 to 6, and the derived parameters are given in Table 4 . For each cloud component there is a range of $b$ and $N$ values that will give an acceptable fit and this is also given in Table 4. For the unsaturated lines, this range of parameters represents the freedom allowed by noise in the data. For saturated lines there is, in principle, no unique solution for $b$ and $N$ within the specified range and a given line can be fitted either with small $b$ and large $N$ or vice versa. The possible range of values is then constrained on one hand by the minimum value of $N$ that will reproduce the observed line (for any value of $b$ ), and, on the other, by the absence of damping wings, which gives quite a precise upper limit to $N$. The possible range of $N$ found in this way can span two orders of magnitude and is of limited astrophysical value.

The formal error in the placement of the continuum is approximately equal to the RMS scatter divided by the square root of the number of points used to define it (Howarth and Phillips 1986). For the present data the continuum error defined in this way was found to have a negligible effect on the column densities. Specifically, for all values of $N$ larger than $10^{12} \mathrm{~cm}^{-2}$ the error was $\leq 1 \%$, and was only $5 \%$ for $N$ values of $5 \times 10^{10} \mathrm{~cm}^{-2}$. These are much smaller than other uncertainties and have been neglected. The error on the zero level has been assumed to be zero for the $\mathrm{Na}$ I observations since it is well defined by the saturated cores of the strong lines. For $\mathrm{Ca}$ II a possible $4 \%$ error was allowed for in the zero level (see $\S$ II, above). This gave corresponding errors in $N$ of between $5 \%$ and $10 \%$ (in the sense of a larger percentage error for stronger lines), and the $\Delta N$ values given for $\mathrm{Ca}$ II in Table 4 allow for these possible errors.

Because of the convolution with the IRF, a $b$ value that is significantly smaller than that of the IRF cannot be determined reliably from the observations. In practice it was found that the models became insensitive to adopted velocity dispersions less than $0.8 \mathrm{~km} \mathrm{~s}^{-1}$ : smaller $b$ values just reproduced the IRF. For this reason many of the narrowest lines only have upper limits for the range of $b$ that are allowed by the data. For some of these lines the actual $b$ value could be $\ll 0.8 \mathrm{~km} \mathrm{~s}^{-1}$. The work of Wayte, Wynne-Jones, and Blades (1978), Blades, WynneJones, and Wayte (1980) and Pettini and Gillingham (1987) has shown that unsaturated $\mathrm{Na}$ I absorption components often have $b$ values of $\leq 0.4 \mathrm{~km} \mathrm{~s}^{-1}$.

For the broad, heavily saturated, lines close to zero velocity, the data were fitted with the minimum possible number of components that would give a satisfactory fit. For $\mathrm{Na}$, this was typically one component. For Ca II, where the lines do not reach zero intensity, it was necessary to use two or three components. Of course, these broad lines are likely to be unresolved blends of much narrower lines, and Hobbs (1974), using 

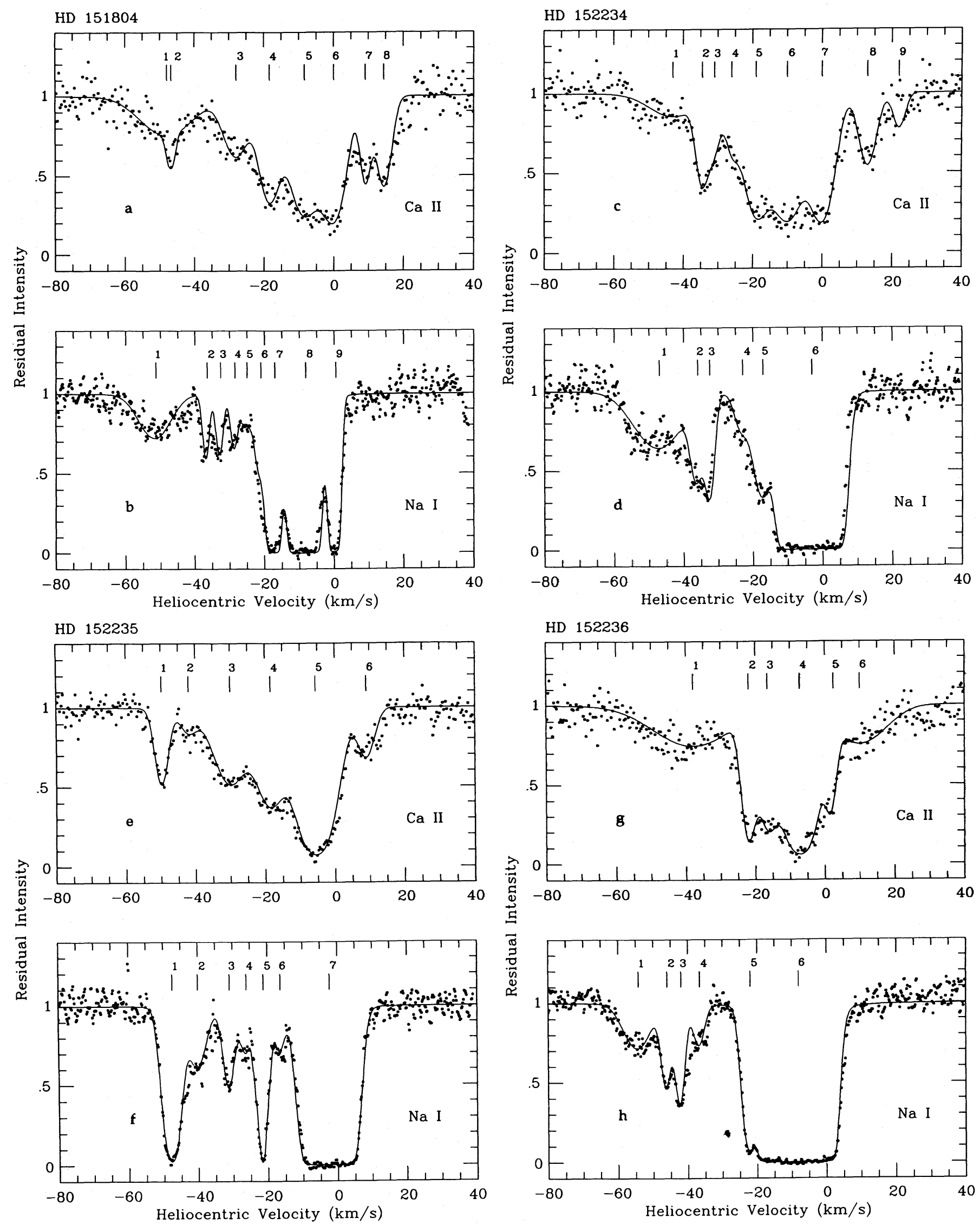

Fig. 2.-The $\mathrm{Ca}$ II and $\mathrm{Na}$ I spectra for the stars observed in both species. The dots are observed intensities, and the solid line is the model fit obtained with the parameters given in Table 4. Identified absorption components are indicated. See text (§ III $a$ ) for details. Note that, for reasons given in the notes to Table 4, the velocity scale for Fig. 20 is approximate only. 

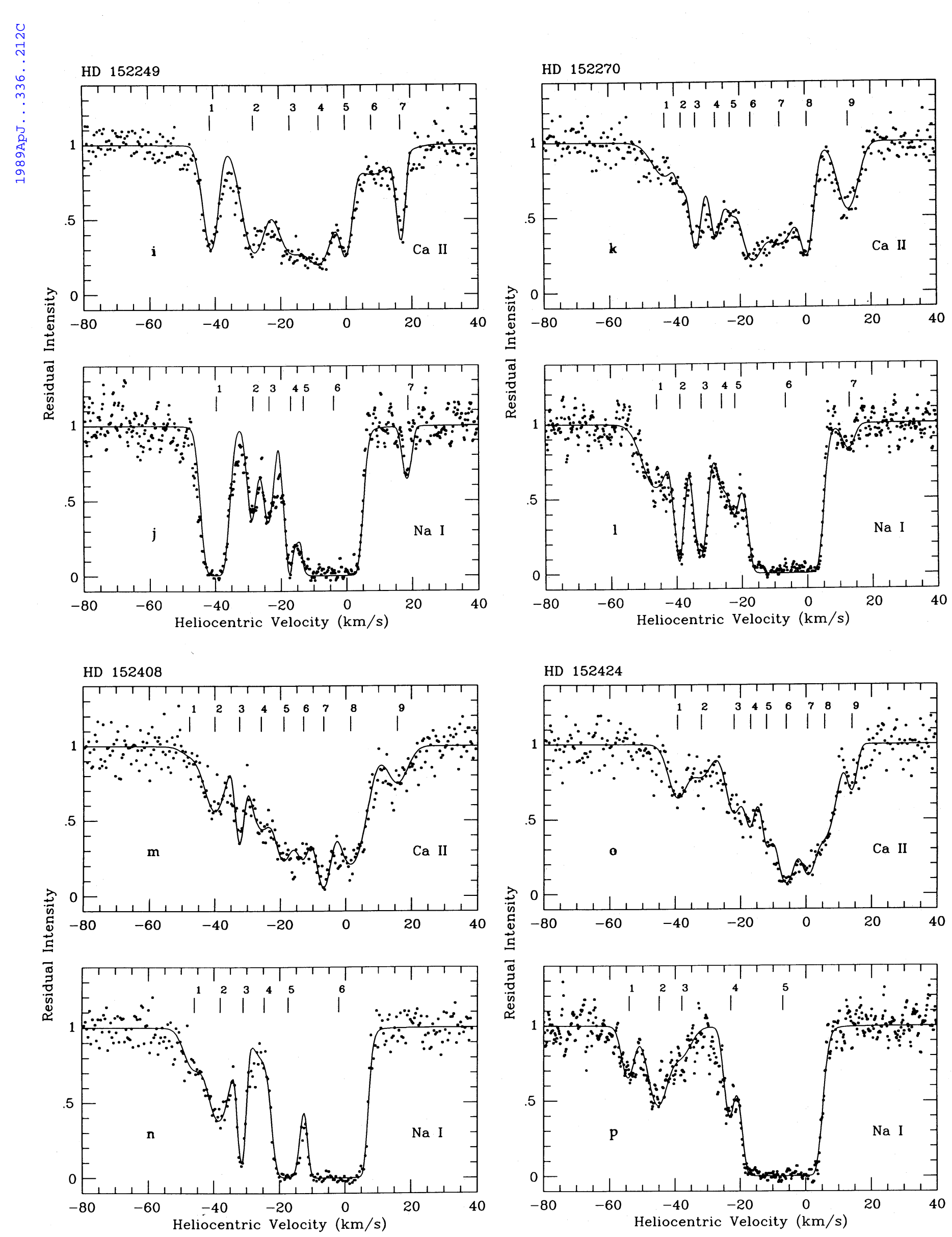

Fig. 2-Continued 

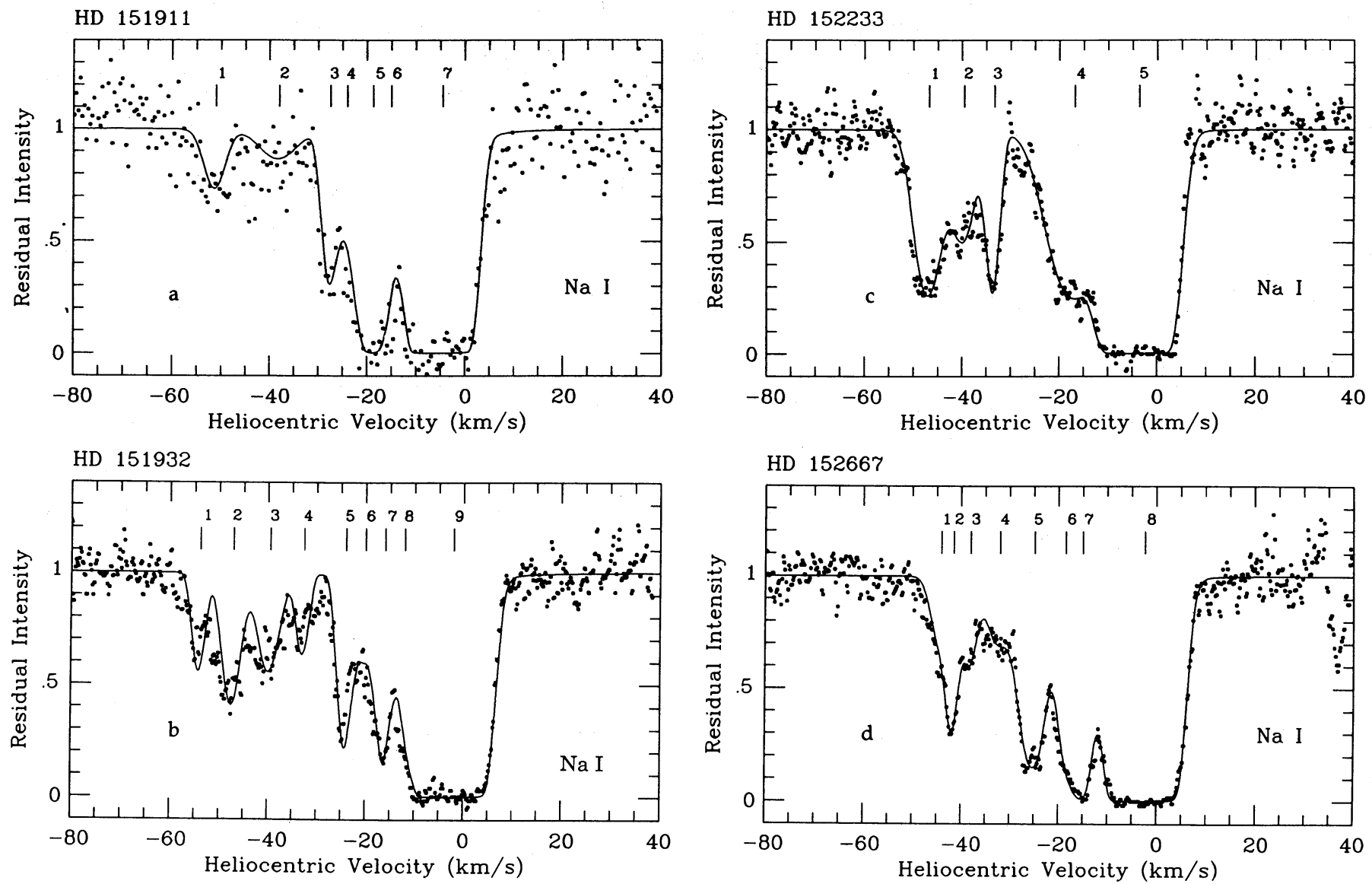

Fig. 3.-The Na I spectra for Sco OB1 association members not observed in Ca II. Details as for Fig. 2. Model parameters are given in Table 4. The apparent absorption feature at about $+35 \mathrm{~km} \mathrm{~s}^{-1}$ in Fig. $3 d$ is due to a mark on the detector.

the unsaturated $K$ I line at $7699 \AA$, found such structure within the saturated $\mathrm{Na}$ I absorption toward several stars. Using the minimum possible number of components gives a lower limit to the column density, but fitting these broad lines with multiple narrow components in selected cases indicated that the column density will still lie within the range quoted in Table 4.

Sometimes it was found to be necessary to introduce components for which there is little or no direct evidence in the spectra, but which were necessary if the observed profile was to be accurately fitted. Thus their existence is implied by the data,

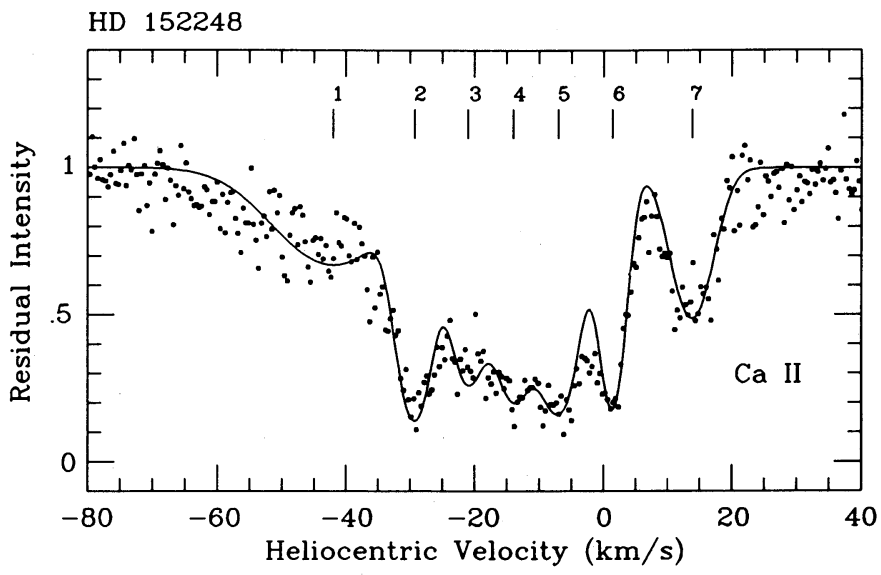

FIG. 4.-The Ca II spectrum for HD 152248 but their $v, b$, and $N$ values are uncertain. Such components are indicated in Table 4 by an asterisk.

Each velocity component is identified by a number in Table 4 which identifies it in Figures 2-6. Since in general different numbers of components were identified for each ion, these numbers are independent and do not correspond between the two species. The velocities given are those determined from the wavelength calibrations discussed in $\S$ II, and those for Ca II are considered to be somewhat more reliable than those for $\mathrm{Na}$ I. Velocity differences of less than about $3 \mathrm{~km} \mathrm{~s}^{-1}$ should not

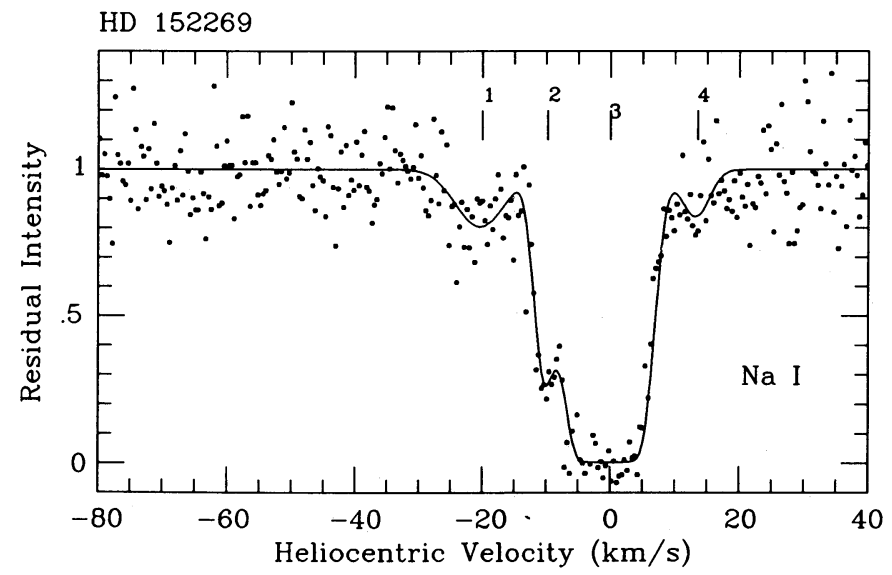

Fig. 5.-The $\mathrm{Na}$ I spectrum toward the foreground star, HD 152269 

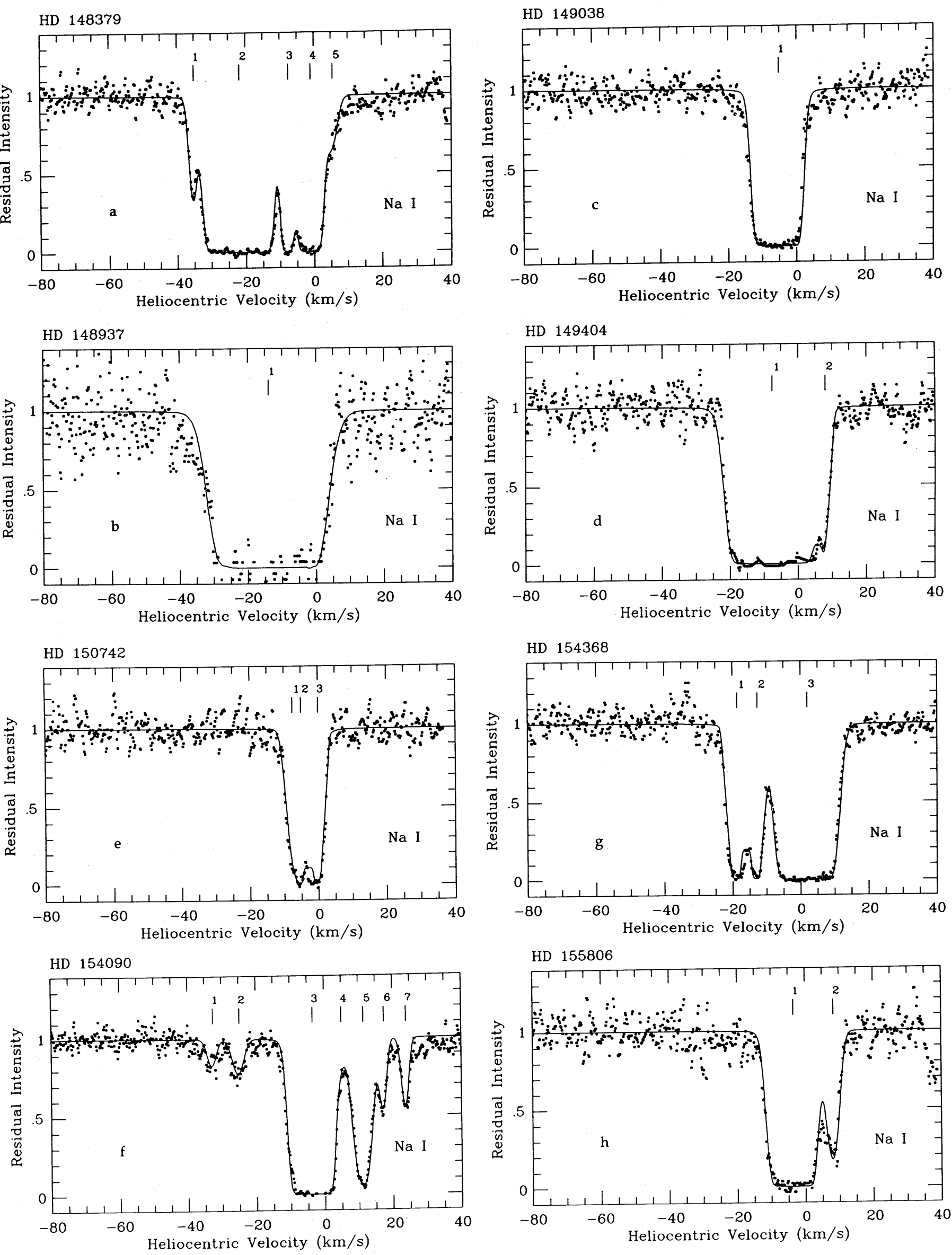

FIG. 6.-The Na I spectra toward the stars outside Sco OB1 
TABLE 4

Model Parameters Adopted for Observed Velocity COMPONENTS

\begin{tabular}{|c|c|c|c|c|c|c|c|}
\hline $\begin{array}{l}\text { Star } \\
\text { HD }\end{array}$ & Ion & $\begin{array}{l}\text { Comp. } \\
\text { No. }{ }^{a}\end{array}$ & $\begin{array}{c}v^{b} \\
\mathbf{k m} \mathbf{s}^{-1}\end{array}$ & $\begin{array}{c}b \\
\mathrm{~km} \mathrm{~s}^{-1}\end{array}$ & 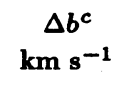 & $\begin{array}{c}\mathrm{N} \\
\mathrm{cm}^{-2}\end{array}$ & $\begin{array}{l}\Delta \mathrm{N}^{d} \\
\mathrm{~cm}^{-2}\end{array}$ \\
\hline 148379 & $\mathrm{Na} \mathrm{I}$ & 1 & -35.1 & 1.0 & $0.8-1.5$ & $2.6(11)$ & $2.0-3.0(11)$ \\
\hline$"$ & $"$ & 2 & -21.8 & 4.5 & $3.5-6.0$ & $2.0(14)$ & $0.3-20.0(14)$ \\
\hline$"$ & $" \prime$ & 3 & -7.6 & 1.0 & $1.0-1.5$ & $6.0(12)$ & $3.0-10.0(12)$ \\
\hline$"$ & $" \prime$ & 4 & -1.0 & 2.3 & $1.8-3.0$ & $1.0(13)$ & $0.3-4.0(13)$ \\
\hline$\prime \prime$ & $\prime \prime$ & $5^{*}$ & +5.5 & 2.0 & $1.5-3.0$ & $1.6(11)$ & $1.4-2.0(11)$ \\
\hline 148937 & $\mathrm{NaI}$ & 1 & -14.0 & 8.5 & $6.0-10.0$ & $1.0(14)$ & $0.4-30(14)$ \\
\hline 149038 & $\mathrm{Na} \mathrm{I}$ & 1 & -5.2 & 3.0 & $2.4-4.0$ & $3.0(14)$ & $0.3-30(14)$ \\
\hline 149404 & $\mathrm{Na} \mathrm{I}$ & 1 & -7.5 & 5.5 & $5.0-6.0$ & $4.0(14)$ & $1.0-7.0(14)$ \\
\hline$"$ & $" \prime$ & 2 & +8.0 & 1.0 & $\leq 1.5$ & $1.0(12)$ & $1.0-1.3(12)$ \\
\hline 150742 & $\mathrm{Na} \mathrm{I}$ & $1^{*}$ & -7.5 & 1.5 & $1.0-1.5$ & $5.0(11)$ & $4.0-6.0(11)$ \\
\hline$"$ & $"$ & 2 & -5.0 & 0.9 & $\leq 1.0$ & $1.0(13)$ & $0.8-5.0(13)$ \\
\hline$\prime \prime$ & $" 1$ & 3 & 0.0 & 0.8 & $\leq 1.0$ & $6.0(13)$ & $3.0-10.0(13)$ \\
\hline 151804 & $\mathrm{NaI}$ & 1 & -51.3 & 6.0 & $5.0-8.0$ & $3.5(11)$ & $2.8-4.0(11)$ \\
\hline " & $"$ & 2 & -36.5 & 0.8 & $\leq 1.3$ & $1.2(11)$ & $1.0-1.4(11)$ \\
\hline$"$ & $"$ & 3 & -32.6 & 1.0 & $\leq 1.3$ & $1.2(11)$ & $1.0-1.4(11)$ \\
\hline$"$ & $"$ & 4 & -28.5 & 0.8 & $\leq 1.3$ & $1.0(11)$ & $1.0-1.2(11)$ \\
\hline$\prime \prime$ & $\prime \prime$ & $5^{*}$ & -25.0 & 3.0 & & $1.2(11)$ & $1.0-1.4(11)$ \\
\hline$"$ & $" \prime$ & $6^{*}$ & -21.0 & 1.5 & $1.0-2.0$ & $2.0(11)$ & $2.0-2.5(11)$ \\
\hline$"$ & $"$ & 7 & -17.0 & 1.4 & $1.2-2.0$ & $5.0(12)$ & $2.0-10.0(12)$ \\
\hline$"$ & $"$ & 8 & -8.2 & 2.3 & $1.8-3.0$ & $7.0(13)$ & $0.7-40.0(13)$ \\
\hline$"$ & $"$ & 9 & +0.5 & 1.0 & $1.0-1.7$ & $6.0(12)$ & $2.0-10.0(12)$ \\
\hline$" \prime$ & $\mathrm{Ca}$ II & 1 & -48.8 & 10.0 & $6.0-13.0$ & $7.0(11)$ & $6.0-8.8(11)$ \\
\hline$"$ & 11 & 2 & -46.8 & 0.9 & $\leq 1.5$ & $1.5(11)$ & $1.0-1.9(11)$ \\
\hline$"$ & $"$ & 3 & -28.0 & 4.5 & $\overline{4.3}-5.5$ & $5.5(11)$ & $4.3-6.1(11)$ \\
\hline$"$ & $" \prime$ & 4 & -18.4 & 3.5 & $3.3-4.5$ & $1.0(12)$ & $0.9-1.2(12)$ \\
\hline$"$ & $"$ & 5 & -8.4 & 5.0 & $5.0-6.0$ & $1.8(12)$ & $1.7-2.1(12)$ \\
\hline$"$ & $" \prime$ & 6 & -0.0 & 4.0 & $3.8-5.0$ & $1.6(12)$ & $1.4-2.1(12)$ \\
\hline$"$ & $"$ & 7 & +9.1 & 1.5 & $1.3-2.0$ & $4.0(11)$ & $2.5-3.3(11)$ \\
\hline$"$ & $" \prime$ & 8 & +14.4 & 2.5 & $2.2-3.0$ & $6.0(11)$ & $5.2-7.7(11)$ \\
\hline 151911 & $\mathrm{NaI}$ & 1 & -51.0 & 2.5 & $1.5-4.0$ & $1.6(11)$ & $1.0-2.5(11)$ \\
\hline$" \prime$ & $" \prime$ & 2 & -38.0 & 5.0 & $4.0-7.0$ & $1.3(11)$ & $0.8-2.5(11)$ \\
\hline$"$ & $"$ & 3 & -27.5 & 1.2 & $0.8-2.0$ & $4.0(11)$ & $4.0-6.0(11)$ \\
\hline$"$ & $" 1$ & $4^{*}$ & -24.0 & 2.0 & $1.0-3.0$ & $2.5(11)$ & $1.0-5.0(11)$ \\
\hline$" 1$ & $"$ & 5 & -18.7 & 2.0 & $1.5-3.0$ & $5.0(12)$ & $3.0-500(12)$ \\
\hline$"$ & $"$ & $6^{*}$ & -15.0 & 1.5 & $\leq 2.5$ & $3.0(11)$ & $1.0-4.0(11)$ \\
\hline " & $"$ & 7 & -4.5 & 3.0 & $2.5-4.5$ & $5.0(14)$ & $0.3-15.0(14)$ \\
\hline 151932 & $\mathrm{Na} \mathrm{I}$ & 1 & -53.8 & 0.9 & $\leq 1.5$ & $2.0(11)$ & $1.3-2.2(11)$ \\
\hline " & $"$ & 2 & -47.1 & 2.0 & $2.0-3.0$ & $4.0(11)$ & $3.0-4.8(11)$ \\
\hline " & $"$ & 3 & -39.5 & 2.5 & $2.5-3.5$ & $3.0(11)$ & $2.5-3.5(11)$ \\
\hline$"$ & $"$ & 4 & -32.6 & 0.8 & $\leq 1.0$ & $1.5(11)$ & $1.0-1.7(11)$ \\
\hline$\prime \prime$ & $"$ & 5 & -24.0 & 1.0 & $\leq 1.0$ & $5.5(11)$ & $5.5-8.0(11)$ \\
\hline$" \prime$ & $"$ & $6^{*}$ & -20.0 & 2.5 & $1 . \overline{5}-3.5$ & $2.5(11)$ & $2.0-2.8(11)$ \\
\hline$"$ & $"$ & 7 & -16.0 & 1.0 & $1.0-2.0$ & $7.0(11)$ & $6.0-8.0(11)$ \\
\hline$"$ & $" 1$ & $8^{*}$ & -12.0 & 1.5 & $1.0-4.0$ & $3.0(11)$ & $2.0-7.0(11)$ \\
\hline$"$ & $"$ & 9 & -2.0 & 3.5 & $3.2-4.0$ & $3.0(14)$ & $0.6-8.0(14)$ \\
\hline
\end{tabular}


TABLE 4-Continued

\begin{tabular}{|c|c|c|c|c|c|c|c|}
\hline $\begin{array}{l}\text { Star } \\
\text { HD }\end{array}$ & Ion & $\begin{array}{c}\text { Comp. } \\
\text { No. }\end{array}$ & $\mathrm{km} \mathrm{s}^{-1}$ & $\begin{array}{c}b \\
\mathrm{~km} \mathrm{~s}^{-1}\end{array}$ & 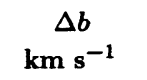 & $\begin{array}{c}\mathrm{N} \\
\mathrm{cm}^{-2}\end{array}$ & $\begin{array}{l}\Delta \mathrm{N} \\
\mathrm{cm}^{-2}\end{array}$ \\
\hline 152233 & $\mathrm{NaI}$ & 1 & -46.7 & 3.0 & $2.8-4.0$ & $8.0(11)$ & $6.5-10.0(11)$ \\
\hline$" \prime$ & $"$ & 2 & -39.5 & 3.0 & $3.0-4.0$ & $4.0(11)$ & $3.0-4.0(11)$ \\
\hline$"$ & $"$ & 3 & -33.3 & 1.0 & $0.8-2.0$ & $4.8(11)$ & $4.8-6.0(11)$ \\
\hline$" \prime$ & $\prime \prime$ & 4 & -16.8 & 6.0 & $5.5-7.0$ & $1.5(12)$ & $1.3-1.8(12)$ \\
\hline$"$ & $"$ & 5 & -3.6 & 4.0 & $3.5-4.3$ & $1.0(14)$ & $0.5-5.0(14)$ \\
\hline 152234 & $\mathrm{NaI}$ & 1 & -47.0 & 9.0 & $7.0-11.0$ & $7.0(11)$ & $6.0-8.0(11)$ \\
\hline$" \prime$ & $"$ & 2 & -36.0 & 2.0 & $1.5-2.5$ & $3.0(11)$ & $2.7-3.4(11)$ \\
\hline$"$ & $" \prime$ & 3 & -32.2 & 1.5 & $1.0-2.0$ & $3.5(11)$ & $3.0-3.8(11)$ \\
\hline$"$ & $"$ & $4^{*}$ & -23.0 & 2.5 & & $1.3(11)$ & $1.0-1.6(11)$ \\
\hline$\prime \prime$ & $" \prime$ & 5 & -17.1 & 3.0 & $2.5-4.0$ & $6.0(11)$ & $5.0-7.0(11)$ \\
\hline$"$ & $" \prime$ & 6 & -3.0 & 4.5 & $4.0-5.0$ & $2.0(14)$ & $0.6-5.0(14)$ \\
\hline$"$ & Ca II & 1 & -43.0 & 9.0 & $7.0-11.0$ & $3.5(11)$ & $3.0-4.7(11)$ \\
\hline$"$ & $"$ & 2 & -34.5 & 2.0 & $1.5-3.0$ & $5.0(11)$ & $4.5-8.8(11)$ \\
\hline$\prime \prime$ & " & $3^{*}$ & -31.0 & 1.5 & $1.0-1.5$ & $2.0(11)$ & $1.0-2.8(11)$ \\
\hline$" \prime$ & $"$ & $4^{*}$ & -26.0 & 2.5 & $1.5-3.5$ & $3.0(11)$ & $2.5-3.9(11)$ \\
\hline$"$ & $"$ & 5 & -19.0 & 4.0 & $3.5-5.0$ & $1.5(12)$ & $1.4-1.7(12)$ \\
\hline " & $" \prime$ & 6 & -10.0 & 5.5 & $5.3-6.5$ & $2.3(12)$ & $2.0-2.8(12)$ \\
\hline$" \prime$ & $"$ & 7 & -0.0 & 4.0 & $3.5-5.0$ & $1.7(12)$ & $1.5-2.0(12)$ \\
\hline$"$ & $"$ & 8 & +13.0 & 3.0 & $2.0-4.0$ & $5.0(11)$ & $3.5-5.8(11)$ \\
\hline " & " & 9 & +22.0 & 2.0 & $1.0-3.0$ & $1.5(11)$ & $1.2-1.9(11)$ \\
\hline 152235 & $\mathrm{Na} I$ & 1 & -47.4 & 2.2 & $1.8-2.5$ & $2.0(12)$ & $1.6-2.5(12)$ \\
\hline$" \prime$ & $"$ & 2 & -40.0 & 3.2 & $2.8-4.0$ & $3.0(11)$ & $2.8-3.5(11)$ \\
\hline$"$ & $"$ & 3 & -30.9 & 1.5 & $1.0-2.0$ & $2.8(11)$ & $2.5-3.2(11)$ \\
\hline$"$ & $"$ & $4^{*}$ & -26.0 & 1.5 & $1.0-2.0$ & $1.3(11)$ & $1.0-1.6(11)$ \\
\hline$" \prime$ & $" \prime$ & 5 & -21.2 & 0.8 & $\leq 1.1$ & $5.0(12)$ & $2.0-100(12)$ \\
\hline$"$ & $"$ & $6^{*}$ & -16.3 & 1.5 & $1.0-2.0$ & $1.2(11)$ & $0.9-1.5(11)$ \\
\hline$"$ & " & 7 & -2.2 & 4.0 & $3.0-5.0$ & $5.0(14)$ & $0.3-20(14)$ \\
\hline " & Ca II & 1 & -49.8 & 2.2 & $1.5-2.8$ & $4.2(11)$ & $3.8-5.3(11)$ \\
\hline$" \prime$ & 11 & $2^{*}$ & -42.0 & 3.2 & $2.5-4.0$ & $1.4(11)$ & $1.3-1.7(11)$ \\
\hline$" \prime$ & $"$ & 3 & -30.0 & 6.0 & $5.0-8.0$ & $1.0(12)$ & $0.8-1.3(12)$ \\
\hline$\prime \prime$ & $"$ & 4 & -18.5 & 5.0 & $4.5-6.0$ & $1.2(12)$ & $1.1-1.5(12)$ \\
\hline$" \prime$ & $"$ & 5 & -5.6 & 6.0 & $5.3-7.0$ & $4.0(12)$ & $4.0-5.0(12)$ \\
\hline$"$ & " & 6 & +9.0 & 3.0 & $2.5-4.0$ & $3.0(11)$ & $2.5-4.0(11)$ \\
\hline 152236 & $\mathrm{Na} I$ & 1 & -54.2 & 5.0 & $4.0-7.0$ & $3.0(11)$ & $2.6-4.0(11)$ \\
\hline$"$ & $"$ & 2 & -46.0 & 1.5 & $1.3-2.0$ & $2.3(11)$ & $1.9-2.5(11)$ \\
\hline$"$ & " & 3 & -42.0 & 1.5 & $1.3-2.0$ & $3.2(11)$ & $2.8-3.4(11)$ \\
\hline$"$ & " & 4 & -36.6 & 2.0 & $1.6-3.0$ & $1.2(11)$ & $1.0-1.5(11)$ \\
\hline$" \prime$ & $" \prime$ & 5 & -22.0 & 2.0 & $1.5-2.2$ & $1.0(12)$ & $0.8-1.2(12)$ \\
\hline$"$ & $" 1$ & 6 & -8.0 & 4.5 & $4.2-6.0$ & $1.0(15)$ & $0.07-2.0(15)$ \\
\hline$"$ & Ca II & 1 & -38.0 & 15.0 & $13.0-18.0$ & $1.1(12)$ & $0.9-1.4(12)$ \\
\hline$"$ & $" 1$ & 2 & -22.0 & 2.0 & $1.5-3.0$ & $1.1(12)$ & $0.9-1.3(12)$ \\
\hline$"$ & $"$ & 3 & -16.5 & 3.0 & $3.0-5.0$ & $1.1(12)$ & $1.1-1.5(12)$ \\
\hline$"$ & $"$ & 4 & -7.2 & 5.5 & $4.8-6.0$ & $4.0(12)$ & $3.5-5.0(12)$ \\
\hline$"$ & $"$ & 5 & +1.5 & 1.4 & $1.0-1.5$ & $4.0(11)$ & $3.0-4.4(11)$ \\
\hline$"$ & $"$ & 6 & +10.0 & 10.0 & $8.0-13.0$ & $7.0(11)$ & $6.5-10.0(11)$ \\
\hline 152248 & Ca II & 1 & -42.0 & 12.0 & $10.0-14.0$ & $1.2(12)$ & $1.0-1.4(12)$ \\
\hline$"$ & $" 1$ & 2 & -29.3 & 3.0 & $2.5-4.0$ & $1.5(12)$ & $1.3-1.7(12)$ \\
\hline$"$ & " & 3 & -21.0 & 3.5 & $3.0-4.5$ & $1.2(12)$ & $1.0-1.4(12)$ \\
\hline$"$ & $\prime \prime$ & 4 & -14.0 & 3.3 & $3.0-4.3$ & $1.3(12)$ & $1.1-1.5(12)$ \\
\hline$"$ & $"$ & 5 & -7.0 & 3.8 & $3.3-4.5$ & $1.8(12)$ & $1.6-1.9(12)$ \\
\hline$"$ & $"$ & 6 & +1.4 & 2.2 & $1.8-3.0$ & $1.1(12)$ & $0.9-1.4(12)$ \\
\hline$"$ & $"$ & 7 & +13.8 & 4.0 & $3.0-5.5$ & $7.5(11)$ & $5.0-10.0(11)$ \\
\hline
\end{tabular}


TABLE 4-Continued

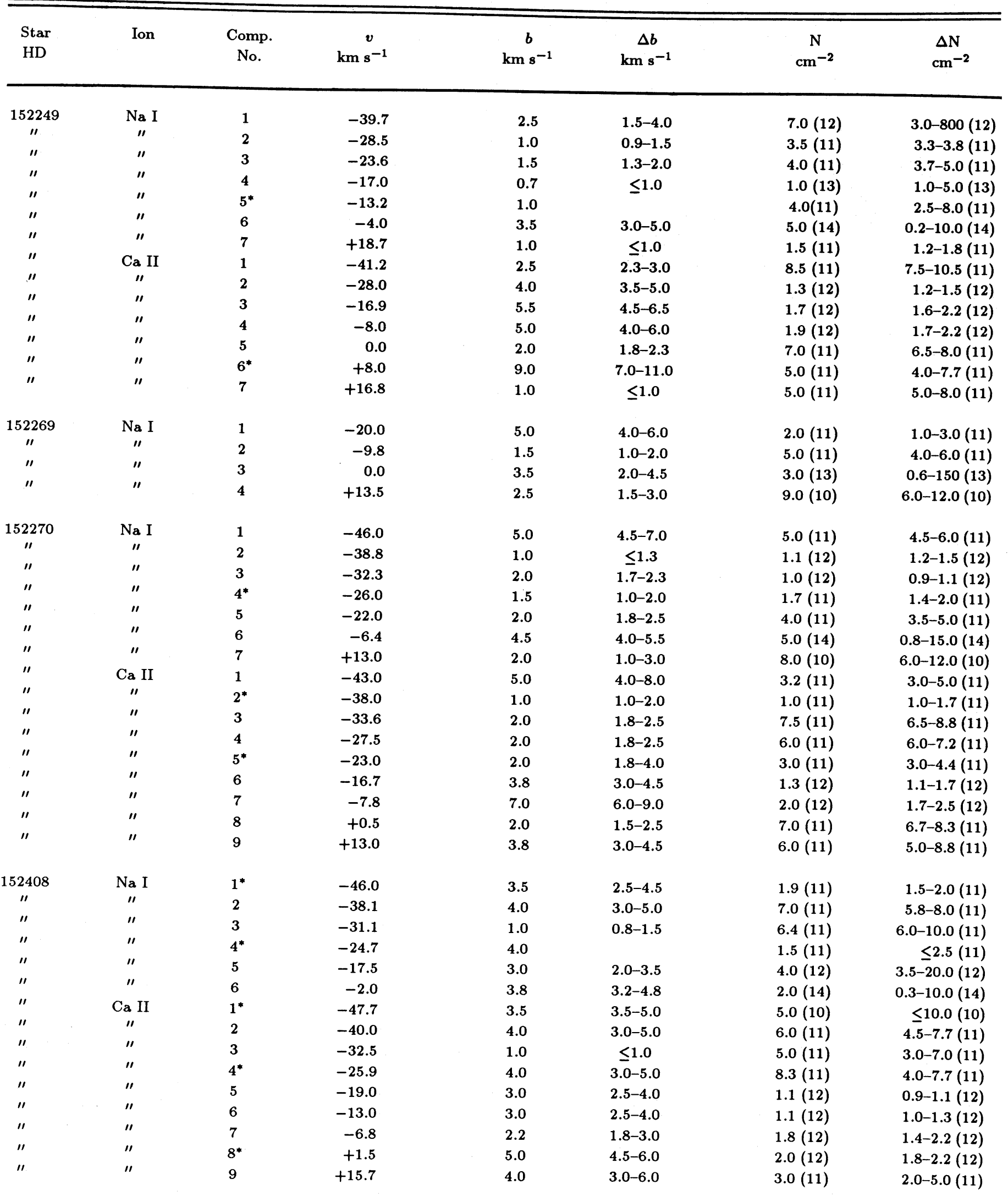


TABLE 4-Continued

\begin{tabular}{|c|c|c|c|c|c|c|c|}
\hline $\begin{array}{l}\text { Star } \\
\text { HD }\end{array}$ & Ion & $\begin{array}{c}\text { Comp. } \\
\text { No. }\end{array}$ & $\mathbf{k m ~ s}^{-1}$ & $\begin{array}{c}b \\
\mathrm{~km} \mathrm{~s}^{-1}\end{array}$ & $\begin{array}{c}\Delta b \\
\mathrm{~km} \mathrm{~s}^{-1}\end{array}$ & $\begin{array}{c}\mathrm{N} \\
\mathrm{cm}^{-2}\end{array}$ & $\begin{array}{c}\Delta \mathrm{N} \\
\mathrm{cm}^{-2}\end{array}$ \\
\hline 152424 & $\mathrm{Na} \mathrm{I}$ & 1 & -54.1 & 2.0 & $1.5-3.0$ & $1.9(11)$ & $1.5-2.5(11)$ \\
\hline$"$ & $"$ & 2 & -45.0 & 3.5 & $2.5-4.0$ & $5.0(11)$ & $4.0-5.6(11)$ \\
\hline$\prime \prime$ & $"$ & $3^{*}$ & -38.0 & 4.0 & $3.0-5.0$ & $1.7(11)$ & $1.3-2.5(11)$ \\
\hline$"$ & $"$ & 4 & -23.0 & 1.5 & $1.0-3.0$ & $3.5(11)$ & $3.0-4.8(11)$ \\
\hline$"$ & $"$ & 5 & -7.0 & 4.2 & $4.2-6.0$ & $5.0(14)$ & $0.6-42.0(14)$ \\
\hline$"$ & $\mathrm{Ca}$ II & 1 & -39.3 & 3.5 & $3.0-7.0$ & $4.0(11)$ & $3.0-5.5(11)$ \\
\hline$"$ & $" \prime$ & $2^{*}$ & -32.0 & 4.0 & $3.0-5.0$ & $2.5(11)$ & $2.0-3.3(11)$ \\
\hline$"$ & $"$ & $3^{*}$ & -22.0 & 3.0 & $2.5-4.0$ & $5.0(11)$ & $4.0-6.6(11)$ \\
\hline$" \prime$ & $"$ & 4 & -16.9 & 1.8 & $1.5-2.5$ & $4.2(11)$ & $4.0-5.0(11)$ \\
\hline$" \prime$ & $"$ & $5^{*}$ & -12.0 & 1.8 & $1.5-2.5$ & $6.0(11)$ & $6.0-7.2(11)$ \\
\hline$" \prime$ & $"$ & 6 & $-6,0$ & 3.0 & $2.8-4.0$ & $2.2(12)$ & $2.0-2.8(12)$ \\
\hline$"$ & $" \prime$ & 7 & +0.5 & 2.5 & $2.5-3.0$ & $1.3(12)$ & $1.1-1.4(12)$ \\
\hline$"$ & $" \prime$ & $8^{*}$ & +5.7 & 4.0 & $3.0-5.0$ & $1.0(12)$ & $0.8-1.3(12)$ \\
\hline " & $"$ & 9 & +14.0 & 1.5 & $1.0-3.0$ & $1.8(11)$ & $1.0-2.2(11)$ \\
\hline 152667 & $\mathrm{Na} \mathrm{I}$ & $1^{*}$ & -44.0 & 2.0 & $2.0-4.0$ & $1.5(11)$ & $1.5-2.0(11)$ \\
\hline$"$ & $"$ & 2 & -41.5 & 1.0 & $0.8-1.5$ & $3.2(11)$ & $3.2-4.0(11)$ \\
\hline$"$ & $"$ & $3^{*}$ & -38.0 & 1.6 & $1.3-2.5$ & $1.8(11)$ & $1.5-2.0(11)$ \\
\hline$"$ & $\prime \prime$ & $4^{*}$ & -32.0 & 3.0 & $2.5-4.0$ & $2.0(11)$ & $1.8-2.5(11)$ \\
\hline$" \prime$ & " & 5 & -24.9 & 3.0 & $2.8-3.5$ & $1.1(12)$ & $1.0-1.3(12)$ \\
\hline$"$ & $"$ & 6 & -18.5 & 1.5 & $1.2-2.5$ & $4.5(11)$ & $4.2-5.5(11)$ \\
\hline$"$ & $" \prime$ & 7 & -15.0 & 2.0 & $1.8-2.5$ & $2.0(12)$ & $1.8-2.5(12)$ \\
\hline$"$ & $"$ & 8 & -2.3 & 3.8 & $3.0-4.5$ & $1.0(14)$ & $0.2-6.0(14)$ \\
\hline 154090 & $\mathrm{NaI}$ & 1 & -32.6 & 1.5 & $1.0-2.0$ & $6.0(10)$ & $4.0-8.0(10)$ \\
\hline$" \prime$ & $"$ & 2 & -24.9 & 2.0 & $1.5-2.5$ & $1.0(11)$ & $0.8-1.2(11)$ \\
\hline$" \prime$ & $"$ & 3 & -3.4 & 3.0 & $2.5-4.0$ & $1.0(14)$ & $0.2-5.0$ \\
\hline$"$ & $"$ & $4^{*}$ & +5.0 & 2.0 & & $6.0(10)$ & $4.0-10.0(10)$ \\
\hline$"$ & $"$ & 5 & +11.5 & 2.5 & $2.0-2.5$ & $1.4(12)$ & $1.2-1.4(12)$ \\
\hline$"$ & $" \prime$ & 6 & +17.5 & 1.0 & $\leq 1.5$ & $1.5(11)$ & $1.5-2.0(11)$ \\
\hline$"$ & $" 1$ & 7 & +24.0 & 1.0 & $\leq 1.5$ & $1.5(11)$ & $1.5-2.0(11)$ \\
\hline 154368 & $\mathrm{NaI}$ & 1 & -18.7 & 1.1 & $0.9-1.5$ & $2.0(13)$ & $1.0-30(13)$ \\
\hline$"$ & $"$ & 2 & -12.7 & 1.0 & $0.8-1.5$ & $2.0(13)$ & $0.3-10(13)$ \\
\hline$"$ & " & 3 & +2.0 & 4.0 & $3.0-5.0$ & $2.2(14)$ & $0.3-20(14)$ \\
\hline 155806 & $\mathrm{Na} \mathrm{I}$ & 1 & -3.4 & 3.3 & $2.5-4.5$ & $1.0(14)$ & $0.1-15(14)$ \\
\hline " & " & 2 & +8.4 & 1.9 & $1.0-2.2$ & $7.2(11)$ & $5.0-8.0(11)$ \\
\hline
\end{tabular}

Note.-See text for details.

a Components marked with an asterisk are not directly obvious in the spectra but are nevertheless necessary in order to produce an acceptable overall fit. The parameters for these components are therefore more uncertain than for the others.

${ }^{b}$ Heliocentric radial velocities. For the Ca II observations of HD 152424, failure to obtain a Th-Ar comparison spectrum means that the velocity scale is only approximate, and comparison with the $\mathrm{Na}$ I spectrum suggests that about $7 \mathrm{~km} \mathrm{~s}^{-1}$ should be subtracted from the velocities listed here.

The range of $b$ that gives an acceptable fit to the observed profiles. Upper limits are given for very narrow components for which a lower limit could not be determined because of insufficient resolution. This column has been left blank for some of the components marked with an asterisk in the third column.

$d$ Range of column density that is consistent with an acceptable fit. For those components with no recorded value of $\Delta b$, these values are estimates. 
TABLE 5

The $N(\mathrm{Na}$ I $) / N(\mathrm{Ca}$ II) Ratios for Stars With Both Na I AND Ca II OBSERVATIONS

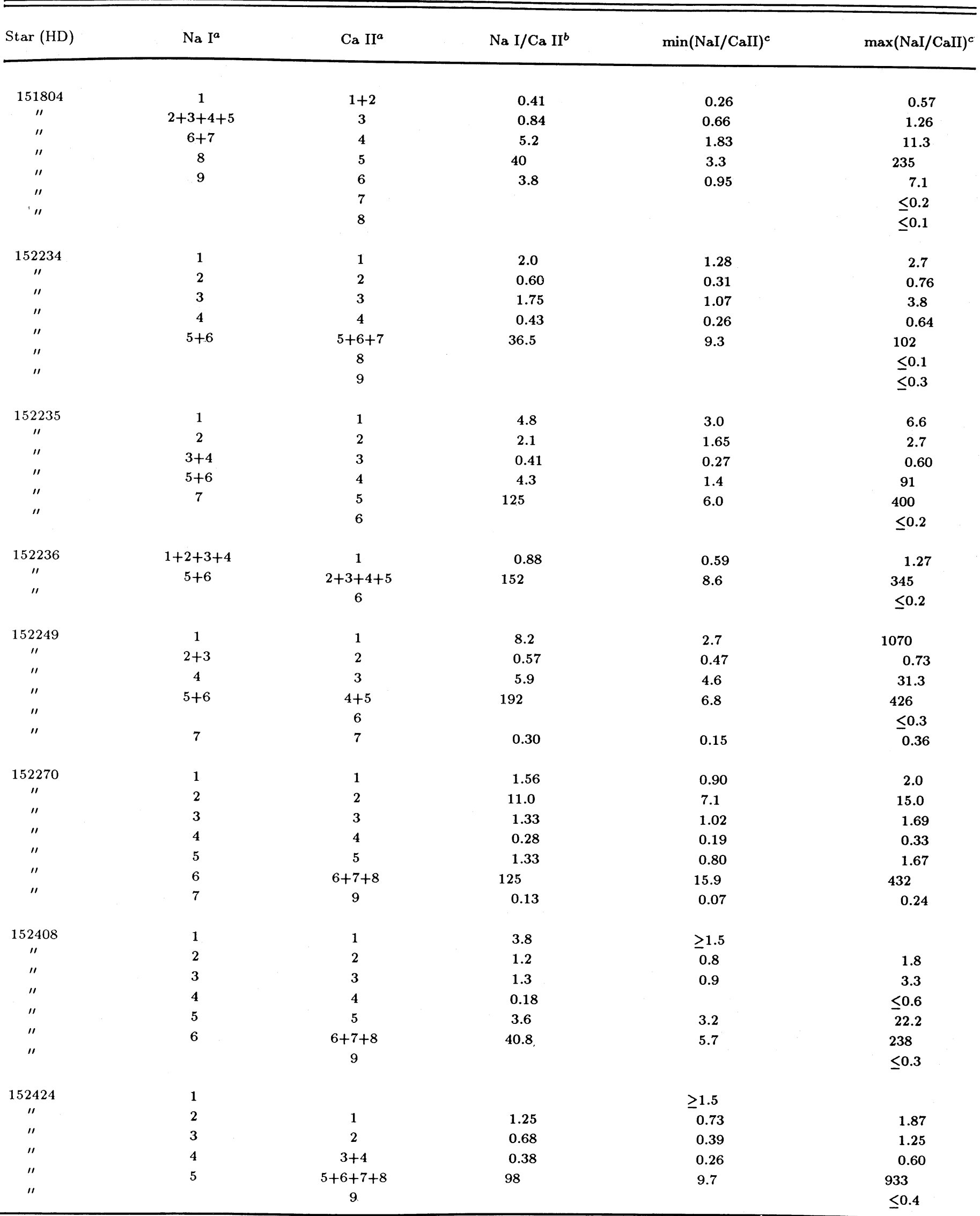


be considered significant. Components in $\mathrm{Na}$ I and $\mathrm{Ca}$ II were judged to correspond if a small $\left(\leq 3 \mathrm{~km} \mathrm{~s}^{-1}\right)$ linear shift could be applied to one spectrum (say, $\mathrm{Na}$ ) to make its velocity components "line up" with components in the other. Whenever the same velocity component could be identified for each ion (not always easy and sometimes not possible), the same $b$ values were used, provided that they gave a satisfactory fit to the data. The allowed range in $b$ was determined independently in each case. Table 5 groups together those components that have been judged to be the same for both $\mathrm{Na}$ I and $\mathrm{Ca}$ II and gives the corresponding $N(\mathrm{Na}$ I $) / N(\mathrm{Ca}$ II) ratios.

\section{b) The $b$ Values}

The identified components can be broadly separated into two groups according to their velocity dispersions; those with $b$ values less than about $2.5 \mathrm{~km} \mathrm{~s}^{-1}$ generally have a symmetrical profile which gives the appearance of being unblended, and those with larger (sometimes much larger) $b$ values are almost certainly the unresolved blends of several components.

It is clear that the broad, fully saturated line seen close to zero velocity in the sodium spectra, although modeled as a single component, is in fact a blend of an unknown number of components. This conclusion is based on the following considerations:

1. Crawford, Rees, and Diego (1987) have resolved three components between +5 and $-15 \mathrm{~km} \mathrm{~s}^{-1}$ in the interstellar $\mathrm{CH}$ line toward HD 152236. In the $\mathrm{Na}$ I spectrum (Fig. $2 h$ ) this velocity range is occupied by a single, saturated feature. An attempt was made to fit this feature with three components with the same velocities and $b$ values as the $\mathrm{CH}$ lines, and with the Na column densities in the same relative proportion as the CH column densities. This reproduced the center and the red wing of the profile very well but did not account for all the absorption near the blue wing, and it is clear that at least one more $\mathrm{Na}$ I component must be present within the feature, at a velocity of about $-17 \mathrm{~km} \mathrm{~s}^{-1}$.

2. The broad saturated absorption trough is partially resolved for several stars, particularly HDs 151804, 148379, and 150742.

3. The Ca II spectra, because they are not fully saturated, cannot be fitted with a single component at this velocity. It was generally found that two or three were necessary as a minimum, and the data are consistent with many more than this.

4. The feature is stronger on the blueward side for more distant stars.

5. The $b$ values necessary to model it as a single component $\left(>3 \mathrm{~km} \mathrm{~s}^{-1}\right)$ are too large for the diffuse cloud material that one would expect to encounter at low velocities (see below).

Many of the spectra also exhibit broad and shallow components with $b$ values between about 5 and $15 \mathrm{~km} \mathrm{~s}^{-1}$, particularly at large negative velocities. We interpret these features as also being due to unresolved blends of sharper lines. Several of these features (for example, component 1 in the sodium spectrum of HD 152234) do show tantalizing hints of substructure, and for HDs 151804 (components 2, 3, and 4) and 151932 (1, 2, 3 , and 4) it was possible to construct plausible models with several components, each with $b \leq 2.5 \mathrm{~km} \mathrm{~s}^{-1}$. There is no doubt that with slightly poorer signal-to-noise ratio these structures would appear as single, broad components.

We turn now to an interpretation of the $b$ values for those components which were well resolved. The velocity spread parameter is a quantitative measure of the velocity dispersion of absorbing atoms within an interstellar cloud. Assuming a Gaussian distribution of velocities, $\psi(v), b$ is equal to $2^{1 / 2}$ times the rms velocity dispersion. Thus

$$
\psi(v)=\frac{1}{b \sqrt{\pi}} \exp \left[\frac{-\left(v-v_{0}\right)^{2}}{b^{2}}\right],
$$

where $v$ is the velocity of a given absorber and $v_{0}$ is the mean velocity, i.e., the bulk velocity of the material.

The value of $b$ for a given cloud is due both to the thermal motion of the atoms within it and to any turbulent motions which may be present. So we have

$$
b=\left(\frac{2 k T}{m}+2 v_{t}^{2}\right)^{1 / 2},
$$

where $v_{t}$ is the rms turbulent velocity along the line of sight, $k$ is Boltzmann's constant, $T$ is the kinetic temperature, and $m$ is the mass of the atom or ion observed.

Since we have no independent way of determining the degree of turbulence within the material, this equation can only be used to deduce upper limits for the kinetic temperature. For the case of no internal turbulence, the $b$-value for sodium is given by

$$
b_{\mathrm{Na}}=0.268(T / 100 \mathrm{~K})^{1 / 2} \mathrm{~km} \mathrm{~s}^{-1} \text {. }
$$

Because of the mass difference between $\mathrm{Na}$ and $\mathrm{Ca}, b_{\mathrm{Na}} / b_{\mathrm{Ca}}=$ 1.32 in the absence of turbulence.

The typical kinetic temperature of interstellar diffuse clouds is generally taken to be about $80 \mathrm{~K}$, from observations of the rotational excitation of molecular hydrogen (e.g., Savage et al. 1977). This corresponds to a $b$ value of only $0.24 \mathrm{~km} \mathrm{~s}^{-1}$, which is far below the resolution of the present study. The very narrowest lines observed had model $b$ values of about $0.8 \mathrm{~km} \mathrm{~s}^{-1}$ and, as explained in $\S$ III $a$, these are themselves upper limits because of the resolution constraint. It follows that those components for which it was not possible to obtain lower limits to $b$ may have temperatures considerably lower than $900 \mathrm{~K}$, and the data would permit diffuse cloud temperatures.

In addition to those which only have upper limits for $b$, many components have well determined upper and lower limits. For example, component 1 at $-47.4 \mathrm{~km} \mathrm{~s}^{-1}$ in the sodium spectrum of HD 152235 (corresponding to component 1 at $-49.8 \mathrm{~km} \mathrm{~s}^{-1}$ in its Ca II spectrum; Figs. $2 e$ and $2 f$, Table 4), has an allowed range of $b$ between 1.8 and $2.5 \mathrm{~km} \mathrm{~s}^{-1}$, which is fairly typical. Since these represent the extremes which will fit the data (for any value of $N$ ), it follows that the combined effect of kinetic temperature and turbulence in this component amounts to an effective temperature (equal to the kinetic temperature in the absence of turbulence) of at least $4.5 \times 10^{3} \mathrm{~K}$, but not more than $8.6 \times 10^{3} \mathrm{~K}$.

\section{Notes TO TABLE 5}

\footnotetext{
a Components in both species that have been identified as belonging to the same velocity component (see text) are grouped together.

${ }^{b} \mathrm{Na}$ I/Ca II ratios obtained from column densities given in Table 4 .

c The minimum and maximum values have been obtained from the extreme values of the column densities given in Table 4. Upper and lower limits are given if a component is seen in one ion but not the other. They are based on the maximum possible strength of the unseen line for a $b$ value equal to that of the identified component.
} 
It is of course not possible to disentangle the contributions of temperature and turbulence, but it is interesting to note that if we assume a kinetic temperature of $80 \mathrm{~K}$ these limits correspond to supersonic velocities, for diffuse cloud conditions, for which the isothermal sound speed is about $0.7 \mathrm{~km} \mathrm{~s}^{-1}$ (Spitzer 1978). Blades, Wynne-Jones, and Wayte (1980) found supersonic turbulent velocities $\left(v_{t}=0.83 \mathrm{~km} \mathrm{~s}^{-1}\right)$ for an interstellar cloud component toward $\eta$ Tau, which they attributed to the effect of the nearby Pleiades stars on the interstellar material.

\section{c) The $\mathrm{Na}$ I/Ca II Ratios}

Figure 7 shows the $\mathrm{Na}$ I/Ca II ratios for all of the components listed in Table 5, as a function velocity. The vertical bars give the range in the ratio allowed by the maximum and minimum column densities found for each component. It is important to realize that these are not error bars in the usual sense; because the $N$ values used to calculate the limits are extreme values based on a wide range of model parameters, we can be almost certain that the actual value of the $\mathrm{Na}$ I/Ca II ratio for each component does lie within the range indicated. In those cases where only one of the ions was identified at a particular velocity, upper or lower limits to the ratio were calculated and have been indicated in the usual way.

One of the components marked in this diagram requires special comment: the very large range indicated for a component at $-40 \mathrm{~km} \mathrm{~s}^{-1}$, which looks out of keeping with its neighbours, is due to the component at this velocity in HD 152249 (Figs. $2 i$ and $2 j$ ). A very large column density for the $\mathrm{Na}$ I component is allowed because the line core reaches zero intensity; however, it can equally well be fitted with a much lower column density, and it is perhaps most likely that the actual value of the $\mathrm{Na} / \mathrm{Ca}$ ratio lies towards the lower limit of its allowed range.

It is immediately obvious from Figure 7 that the $\mathrm{Na}$ I/Ca II ratios can be divided into three velocity bands, the boundaries of which will be used to classify the components. That is, the $\mathrm{Na}$ I/Ca II ratios are significantly different for the positive velocity gas, for the low negative velocity gas, and for the high negative velocity gas. We will return in $\S \mathrm{V}$ to the interpretaion of this velocity dependence, after first discussing in detail, in $\S \mathrm{IV}$, the characteristics of each of these velocity groups.

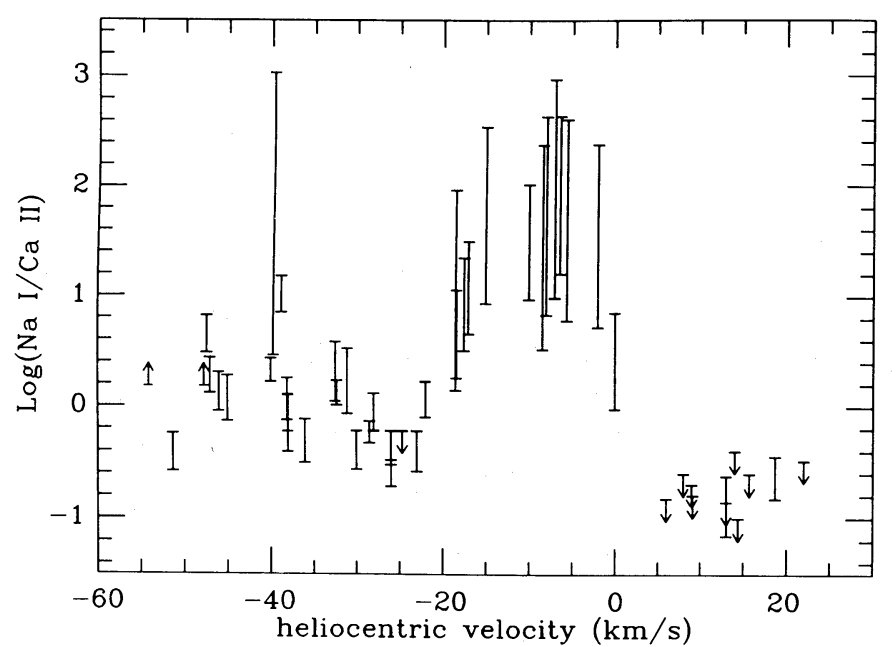

FIG. 7.--Plot showing the observed $N(\mathrm{Na} \mathrm{I}) / N(\mathrm{Ca}$ II) ratio as a function of heliocentric velocity. See text ( $\$$ III $c$ and V) for details and discussion.

\section{THE VELOCITY STRUCTURE}

In addition to the clear differences in the derived $\mathrm{Na}$ I/Ca II ratios seen in Figure 7, the observed $\mathrm{Na}$ I and $\mathrm{Ca}$ II interstellar line spectra show the following noticeable features, as a function of radial velocity:

1. All of the $\mathrm{Na}$ I spectra show fully saturated absorption components at velocities extending from $0 \mathrm{~km} \mathrm{~s}^{-1}$ to at least $-10 \mathrm{~km} \mathrm{~s}^{-1}$, and often to $-20 \mathrm{~km} \mathrm{~s}^{-1}$.

2. All nine of the Ca II spectra, but only four of the $21 \mathrm{Na}$ I spectra, show absorption components at velocities more positive than $+9 \mathrm{~km} \mathrm{~s}^{-1}$.

3. All of the Sco OB1 association members (including NGC 6231) show discrete blue shifted $\mathrm{Na}$ I and $\mathrm{Ca}$ II absorption components at velocities more negative than $-40 \mathrm{~km} \mathrm{~s}^{-1}$. None of the stars in the foreground or outside Sco OB1 show such components.

We will consider each of these three velocity component groups in turn, in $\S \mathrm{IV} b, \operatorname{IV} d$, and $\operatorname{IV} f$, as well as considering the velocity structure expected from Galactic rotation ( $\$ \mathrm{IV} a$ ), and the information provided by the interstellar spectra of the stars in the foreground, or nearby on the sky, to Sco OB1 $(\S \S \mathrm{IV} c$ and IVe).

\section{a) The Velocity Structure Expected from Galactic Rotation}

By adopting a value for the Oort constant $A$ of $14.4 \pm 1.2$ $\mathrm{km} \mathrm{s}^{-1}$ (Kerr and Lynden-Bell 1986), a distance to Sco OB 1 of $1900 \pm 300$ pc (Humphreys 1978) and a mean galactic longitude of 343.5 , one predicts a radial velocity due to Galactic rotation of $-14.9 \pm 2.0 \mathrm{~km} \mathrm{~s}^{-1}$ relative to the local standard of rest. At this Galactic longitude, heliocentric velocities are 5.4 $\mathrm{km} \mathrm{s}^{-1}$ more negative than LSR values, so we would expect a heliocentric radial velocity for Sco OB1 of $-20.3 \pm 2 \mathrm{~km} \mathrm{~s}^{-1}$. Laval (1972) gives an average heliocentric velocity for the association of $-23.9 \pm 1.4 \mathrm{~km} \mathrm{~s}^{-1}$ determined from objective prism spectroscopy. Struve (1944) found $-29.1 \pm 2.4 \mathrm{~km} \mathrm{~s}^{-1}$ from 13 " normal" member stars.

For the present purpose, we will assume that the association is corotating with the Galaxy and that those components with heliocentric velocities less negative than $-25 \mathrm{~km} \mathrm{~s}^{-1}$ (excluding the positive velocities) arise from Galactic rotation. We note, however, that an uncertainty of at about $\pm 5 \mathrm{~km} \mathrm{~s}^{-1}$ exists on this value.

\section{b) The Low-Velocity Gas $\left(-20 \mathrm{~km} \mathrm{~s}^{-1} \leq v_{\text {hel }} \leq 0 \mathrm{~km} \mathrm{~s}^{-1}\right)$}

Absorption components in this velocity range are most likely to be due to material distributed between the observer and Sco OB1, with Galactic rotation producing the observed velocity spread. In the $\mathrm{Na}$ I spectra this velocity range is dominated by a single, fully saturated, component with a heliocentric velocity between about zero and $-10 \mathrm{~km} \mathrm{~s}^{-1}$. This is very close to zero-velocity LSR. The less heavily saturated Ca II data indicate that this component is probably due to several absorbing clouds. Moreover the $b$ values need to fit the $\mathrm{Na}$ I data with one component are much larger than one would expect for a single interstellar cloud ( $($ III $b$ ).

Structure on the blue wing of the main absorption feature (but still in the low-velocity domain) can be seen in most of the spectra. In particular a component at about $-17 \mathrm{~km} \mathrm{~s}^{-1}$ is present in the $\mathrm{Na}$ I spectra toward the following stars: HD $151804\left(-17 \mathrm{~km} \mathrm{~s}^{-1}\right), 151932\left(-15 \mathrm{~km} \mathrm{~s}^{-1}\right), 152233(-17 \mathrm{~km}$ $\left.\mathrm{s}^{-1}\right), 152234\left(-17 \mathrm{~km} \mathrm{~s}^{-1}\right), 152235\left(-16.3 \mathrm{~km} \mathrm{~s}^{-1}\right), 152236$ $\left(\sim-17 \mathrm{~km} \mathrm{~s}^{-1} ; \S\right.$ III $\left.b\right), 152249\left(-17 \mathrm{~km} \mathrm{~s}^{-1}\right), 152408(-17.5$ 
$\left.\mathrm{km} \mathrm{s}^{-1}\right)$, and $152267\left(-18.5\right.$ and $\left.-15 \mathrm{~km} \mathrm{~s}^{-1}\right)$. The absence of this component toward HDs 152270 , and 152424 may be due to blending with the saturated line nearby, as in the case of HD $152236(\S \mathrm{III} b)$. A component at $-16.7 \mathrm{~km} \mathrm{~s}^{-1}$ was identified in the Ca II spectrum of HD 152270 and the Ca II spectra for the other stars are consistent with a component at this velocity. It appears therefore that a component at about $-17 \mathrm{~km} \mathrm{~s}^{-1}$ is common to at least seven of the member stars and could be present for all of them, although its strength varies considerably.

Several stars also have prominent components at about $-22 \mathrm{~km} \mathrm{~s}^{-1}$, e.g., HD $152235\left(-21.2 \mathrm{~km} \mathrm{~s}^{-1}\right), 152236(-22$ $\left.\mathrm{km} \mathrm{s}^{-1}\right), 152270\left(-22 \mathrm{~km} \mathrm{~s}^{-1}\right)$, and $152424\left(-23 \mathrm{~km} \mathrm{~s}^{-1}\right)$. This is sufficiently close to the adopted radial velocity for Sco OB1 for this component to be associated with it. However, the fact that the foreground star HD 152269 also shows an absorption component at $-20 \mathrm{~km} \mathrm{~s}^{-1}$ (Fig. 5 and Table 4) indicates an origin considerably in front of Sco OB1. As discussed in $\S \mathrm{IV} c$, a similar velocity component is seen toward members of the Sco OB2 association, which are only $170 \mathrm{pc}$ distant (Hobbs 1969).

From a study of the reddening undergone by stars in the direction of Sco OB1, Schild, Neugebauer, and Westphal (1971) concluded that most of the reddening to the association stars occurred within $1000 \mathrm{pc}$ of the Sun, in the local spiral arm. In a similar study, van Genderen, Bijeveld, and van Groningen (1984) estimated that most of the reddening occurred within $700 \mathrm{pc}$ of the Sun. It is therefore likely that most of the broad, low-velocity absorption seen towards all of the stars occurs within such a distance scale.

\section{c) The Velocity Structure toward the Foreground Star HD 152269}

HD 152269 was observed because it lies in the same direction as Sco OB1 and because the photometry of Schild, Neugebauer, and Westphal (1971) and of van Genderen, Bijeveld, and van Groningen (1984), along with the abundance analysis of Keenan et al. (1984), support the view that it is a foreground star. Schild et al. estimated a photometric distance of $720 \mathrm{pc}$, based upon their classification of it as a B7III star.

The Na I D2 spectrum of HD 152269 (Fig. 5) does not have any absorption components with velocities more negative than about $-20 \mathrm{~km} \mathrm{~s}^{-1}$. There can be no doubt therefore that the highly blueshifted components observed toward the Sco OB1 stars are produced beyond HD 152269. The broad, weak feature at about $-20 \mathrm{~km} \mathrm{~s}^{-1}$ could be common to all of the stars, but be swamped by the stronger absorptions at this velocity in the other spectra. It is interesting to note that this component is similar to the highest negative velocity component seen in the $\mathrm{Na}$ I spectra of many of the Sco OB2 association stars, which are at a distance of only $170 \mathrm{pc}$ (e.g., the -22 $\mathrm{km} \mathrm{s}^{-1}$ component in the spectrum of $\delta$ Sco; Hobbs 1969).

\section{d) The Positive Velocity Components $\left(v_{\mathrm{hel}} \geq+9 \mathrm{~km} \mathrm{~s}^{-1}\right)$}

All nine of the Ca II spectra, and four of the $21 \mathrm{Na}$ I spectra, show absorption components between about +9 and $+24 \mathrm{~km}$ $\mathrm{s}^{-1}$, i.e., $v_{\mathrm{LSR}}=+14$ to $+29 \mathrm{~km} \mathrm{~s}^{-1}$, well outside the range predicted by the effects of Galactic rotation.

One possible interpretation of these weak components could be that they represent the receding parts of an expanding shell of gas which is intercepted along these sight lines, in which case the components corresponding to the approaching side of the shell could easily be hidden within the strong broad absorption troughs extending between 0 and $-20 \mathrm{~km} \mathrm{~s}^{-1}$.

However, another interpretation of the weak positive velocity components is possible, arising from the fact that $\mathrm{Na} \mathrm{I}$ and Ca II observations of nearby lightly reddened stars often show weak absorption extending to velocities as far as $v_{\mathrm{LSR}}= \pm 20$ $\mathrm{km} \mathrm{s}^{-1}$. For example, the sight line to $\mu^{1} \operatorname{Sco}(D=210 \mathrm{pc})$ shows Ca II absorption out to $v_{\mathrm{LSR}}=-26 \mathrm{~km} \mathrm{~s}^{-1}$ (Hobbs $1984)$ and $\lambda$ Sco $(D=110 \mathrm{pc})$ shows Ca II absorption out to $v_{\mathrm{LSR}}=-20 \mathrm{~km} \mathrm{~s}^{-1}$ (Vidal-Madjar et al. 1986); while the data of Hobbs (1978a) show Ca II absorption extending to $v_{\text {LSR }}+12$ $\mathrm{km} \mathrm{s}^{-1}$ for the case of $\gamma$ Ori $(D=110 \mathrm{pc})$ and to $-12 \mathrm{~km} \mathrm{~s}^{-1}$ for $\alpha$ Vir. These four sight lines have $\mathrm{Na}$ I/Ca II ratios of between 0.4 and 0.7 . The weak $\mathrm{Na}$ I and $\mathrm{Ca}$ II absorption along the longer path lengths to the lightly reddened stars S Mon, $\kappa$ $\mathrm{CMa}, \mathrm{O}^{2} \mathrm{CMa}$, and $\zeta$ Pup show similar velocity extents and $\mathrm{Na}$ I/Ca II ratios. From an analysis of the ratios toward such stars, Hobbs (1978a) and Ferlet, Vidal-Madjar, and Gry (1985) have concluded that the components could arise either in cool gas $(T \sim 80 \mathrm{~K})$ with a low degree of ionization $\left(n_{e} \sim 10^{-3}\right.$ $\left.\mathrm{cm}^{-3}, n_{\mathrm{H}} \sim 3 \mathrm{~cm}^{-3}\right)$, or in warm, partially ionized gas $\left(n_{e} \sim\right.$ $0.04 \mathrm{~cm}^{-3}$ for $T \sim 8000 \mathrm{~K}$ ). The latter alternative could correspond to an intercloud medium. Cowie and York (1978) have found that absorption components due to warm low-density gas possess a significantly broader range of velocities than do cool cloud components.

It may be possible to identify the weak positive velocity components in the present sample with such low-density material. Their derived $b$ values are consistent with thermal broadening at intercloud temperatures, whereas their broadening would have to be by supersonic turbulence for $T \sim 80 \mathrm{~K}$. For the two stars with both $\mathrm{Na} I$ and $\mathrm{Ca}$ II detectable (HD 152249 and $\mathrm{HD} 152270$ ), the derived $\mathrm{Na}$ I/Ca II ratios are 0.3 (limits of $0.15-0.36$ ) and 0.13 (limits of $0.07-0.24$ ), respectively, while for the stars for which only $\mathrm{Ca}$ II was detected at positive velocities, the upper limits to this ratio lie in the range $0.1-0.4$. These ratios are about a factor of 2 smaller than the typical values found for the lightly reddened stars discussed above, although the $\mathrm{Na} \mathrm{I} / \mathrm{Ca}$ II ratio for $\kappa \mathrm{CMa}[E(B-V)=0.02]$ is 0.15 (Hobbs $1978 a, b)$.

It is possible that material similar to that responsible for the weak positive velocity components is distributed all along the line of sight to the Sco OB1 association. If the material can have peculiar velocities of up to $\pm 20 \mathrm{~km} \mathrm{~s}^{-1}$ relative to its surroundings, then weak absorption by such components could be present up to $v_{\text {hel }}=-40 \mathrm{~km} \mathrm{~s}^{-1}$ in the spectra of the Sco OB1 stars. We will return to this point in $\S \mathrm{V}$.

\section{e) The Velocity Structure toward the Stars outside Sco OB1}

Eight stars lie within $9^{\circ}$ of Sco OB1 on the sky (Table 1 and Fig. $1 a$ ) but at a variety of distances, although none is as distant as Sco OB1 itself.

HD 148379, 148937, and 149404 are generally taken to be members of the Ara OB1a association, for which Herbst and Havlen (1977) obtained a distance of $1320 \pm 120 \mathrm{pc}$, while Humphreys (1978) estimated a distance of $1380 \pm 200$ pc. Although they are $2^{\circ}$ apart on the sky (corresponding to a linear separation of $46 \mathrm{pc}$ for a distance of $1350 \mathrm{pc}$ ), the interstellar Na I D2 spectra of HD 148379 and HD 148937 are very similar to each other, with the higher signal-to-noise ratio of the former spectrum allowing more structure to be discerned (observations of the latter star were curtailed by cloud). HD 
148379 shows structure at velocities more negative than -20 $\mathrm{km} \mathrm{s}^{-1}$ (Fig. 6a), although the very broad feature seen toward HD 148937 (Fig. 6b) could easily include features at this velocity.

The peculiar figure-eight-shaped nebula NGC 6164-5 surrounds the luminous Of star HD 148937-this nebulosity has often been assumed to have been caused by ejection events by the star. However, unlike the Sco OB1 stars, no Na I absorption features are seen at velocities more negative than $-35 \mathrm{~km}$ $\mathrm{s}^{-1}$. Although Leitherer and Chavarria-K (1987) found $\mathrm{H} \alpha$ emission components near to HD 148937 with radial velocities of -70 to $-80 \mathrm{~km} \mathrm{~s}^{-1}$, apparently no high-velocity material due to the nebula is intercepted in the sight line to this star.

The spectrum of the BO Ia star HD 149038 ( $\mu$ Nor) is of particular interest since only a narrow $\mathrm{Na}$ I absorption component is seen (Fig. 6c). This star is usually taken to be a member of the Ara OB1a association (e.g., Humphreys 1978) and hence to lie at a distance of about $1.3 \mathrm{kpc}$. Its relative narrow $\mathrm{Na}$ I absorption feature, compared to those of HD 148379 and HD 148937, is therefore hard to understand. An explanation could be provided by the work of Carrasco and Crézé (1978) who suggested that runaway O stars (including HD 149038) might be underluminous by at least 2.5 mag with respect to normal stars of the same spectral type. If this is the case for HD 149038, its distance might be as little as $400 \mathrm{pc}$, providing an explanation for its simple interstellar spectrum.

The $\mathrm{Na}$ I absorption feature observed toward HD 149038 (Fig. 6c) appears to cover the same velocity range as do components 3 and 4 in the spectrum of HD 148379 (Fig. $6 a$ ). Since HD 149038 is reddened by an $E(B-V)$ of only 0.31 , compared to $E(B-V)=0.76$ for HD 148379 , it is clear that the more negative velocity components ( 1 and 2$)$ in the $\mathrm{Na}$ I spectrum of HD 148379 are associated with material beyond HD 149038 having an $E(B-V)$ of about 0.45 .

The Na I feature observed toward HD 149404 (Fig. 6d) extends to larger positive velocities than for either 148379 or 148937 and is not as broad. It appears that for this star $(3.6$, or $87 \mathrm{pc}$, away from HD 148379) has at least one additional positive velocity component, and is missing at least one negative velocity component, compared to the other two Ara OB1a stars.

HD 150742 is the closest "field star" to Sco OB1 on the sky (Fig. 1a) but is relatively close to the sun (estimated distance about $260 \mathrm{pc}$ ). It is useful for it demonstrates conclusively that the very broad, saturated low-velocity components seen toward all stars are made up of a number of discrete components. Three were identified in the line profile analysis and the mismatch that exists between the data and the model (Fig. $6 e$ ) indicates that at least one more component is probably present.

The red wings of the $\mathrm{Na}$ I absorption features toward HD 149038 and HD 150742 coincide, whereas the blue wing of the $\mathrm{Na}$ I feature toward HD 149038 extends to significantly more negative velocities, indicating that at least one more absorption component is present and that HD 149038 is probably the more distant of the two stars.

Both HD 154368 and HD 155806, on the othei side of Sco OB1 as seen on the sky, are members of the Sco OB4 association (Hoffleit and Jaschek 1982) and have a distance of about 900 pc (Table 1). Absorption component 2 toward HD 155806 (Fig. $6 h$ ) coincides with the redward wing of the broad absorption component 3 seen toward HD 154368 (Fig. 6g), demonstrating that the latter component is caused by more than one absorbing system. The most negative absorption seen toward HD 155806 fills the gap seen between components 2 and 3 in the spectrum of HD 154368 , so HD 155806 has no Na I components corresponding to the two most negative velocity components seen toward HD 154368 (components 1 and 2, at -18.7 and $-12.7 \mathrm{~km} \mathrm{~s}^{-1}$, respectively).

HD 155806 is only $2: 7$ away from HD 154368 on the sky (corresponding to about $40 \mathrm{pc}$ for $D=900 \mathrm{pc}$ ) and in addition to lacking the two most negative velocity components seen toward HD 154368 , has an $E(B-V)$ which is 0.45 smaller. It is tempting to think that perhaps its distance has been underestimated, as was suggested for HD 149038. However, since there is nothing unusual about either its radial velocity or its proper motion (Hoffleit and Jaschek 1982), the runaway effect of Carrasco and Crézé (1978) cannot be invoked. Unless the distance has been overestimated for some other reason, its much lower reddening and the lack of absorption features at velocities more negative than $-10 \mathrm{~km} \mathrm{~s}^{-1}$ implies major inhomogeneities in the interstellar medium on scales of $40 \mathrm{pc}$ or less.

This conclusion is supported by the $\mathrm{Na}$ I spectrum for $\mathrm{HD}$ 154090 (Fig. 6f) which has strong positive components not present toward any other star. Note that although component 3 corresponds to component 1 in HD 155806 (Fig. 6h), component 5 does not match with component 2 of the latter star. HD 154090 also has two weak components at -32.6 and $-24.9 \mathrm{~km} \mathrm{~s}^{-1}$ not present in the other "field stars" and similar to the large negative velocity components observed toward the Sco OB1 stars (see below).

\section{f) The Large Negative Velocity Components $\left(v_{\text {hel }} \leq-40 \mathrm{~km} \mathrm{~s}^{-1}\right)$}

The largest negative velocities, observed only toward Sco $\mathrm{OB} 1$, are in the region of -40 to $-60 \mathrm{~km} \mathrm{~s}^{-1}$, much larger than can be accounted for by Galactic rotation. Although the general features of the spectra are similar, significant differences in detail exist which indicate that the blueshifted material is not homogeneous across the association. At the distance of the association, its approximate angular diameter of $2^{\circ}$ corresponds to a linear diameter of $66 \mathrm{pc}$. Particularly interesting are the differences observed towards HD 152233 ( $\mathrm{Na}$ I only), 152234,152248 (Ca II only), 152249, and 152270, which are all members of NGC 6231 and are within $4^{\prime}$ of each other, corresponding to $2 \mathrm{pc}$ for a distance of $1900 \mathrm{pc}$. HD 152233 and HD 152234 are separated by only $1^{\prime}(0.5 \mathrm{pc}$ for $D=1900 \mathrm{pc})$ and yet their $\mathrm{Na}$ I spectra are quite different, particularly shortwards of $-30 \mathrm{~km} \mathrm{~s}^{-1}$ (Figs. $3 c$ and $2 d$, respectively). It is clear that very small scale structure exists in the absorbing material.

Similar blueshifted absorption components have been seen toward other OB associations $(\S I)$, the most extreme example being Car OB1, where negative velocities as large as 180-200 $\mathrm{km} \mathrm{s}^{-1}$ were seen in Ca II by Walborn and Hesser (1975), who also found large variations in the line profiles over small angular scales. Significantly larger interstellar line velocities have been found at ultraviolet wavelengths toward the Carina stars (Walborn and Hesser 1982; Laurent, Paul, and Pettini 1982). This does not seem to be the case for the Sco OB1 stars. Cowie $e t$ al. (1981) report C II absorption at a velocity of -85 $\mathrm{km} \mathrm{s}^{-1}$ toward HD 151804 and $-70 \mathrm{~km} \mathrm{~s}^{-1}$ toward HD 152408. These are only $20-30 \mathrm{~km} \mathrm{~s}^{-1}$ more negative than the most negative velocity components seen in $\mathrm{Na}$ I and $\mathrm{Ca}$ II $(-51$ and $-48 \mathrm{~km} \mathrm{~s}^{-1}$, respectively). Moreover, an examination of $I U E$ archive spectra shows that these $C$ II velocities correspond to the blue edge of broad, fully saturated, absorption troughs. 
It is likely that the highest negative velocity components hidden in these features actually occur at more positive velocities than this edge owing to the convolution with the IUE instrumental response function $\left(\sim 30 \mathrm{~km} \mathrm{~s}^{-1} \mathrm{FWHM}\right)$.

The velocities of the most blueshifted absorption features in each spectrum appear to be about $20-35 \mathrm{~km} \mathrm{~s}^{-1}$ more negative than the radial velocity of -20 to $-25 \mathrm{~km} \mathrm{~s}^{-1}$ estimated for the association $(\S \mathrm{IVa}$ ). It therefore appears simplest to interpret the absorption seen between -40 and $-60 \mathrm{~km} \mathrm{~s}^{-1}$ as arising from material expanding around the Sco OB1 stars.

The interaction between stellar winds and the interstellar medium has been studied by Pikelner (1968), Dyson and de Vries (1972), Avedisova (1972), and subsequent authors. Many of the stars in Sco OB1 are known to have large mass loss rates. However, we find no correlation between estimated mass-loss rates (Bertout et al. 1985) and either the column densities in the large negative velocity components, or their maximum velocities. It therefore appears that the small-scale velocity structure seen toward the Sco OB1 stars is not due to a different swept-up shell around each star, but rather is caused by density and velocity inhomogeneities within a large expanding gas complex around the association.

Bruhweiler et al. (1980) have used the theory of Weaver et al. (1977) to compute the evolution of an expanding swept-up shell around an OB association, for which purpose they took Sco OB1 to represent a typical unevolved example. For a mean ambient interstellar hydrogen number density of $1 \mathrm{~cm}^{-3}$, they predicted an expansion velocity of $21 \mathrm{~km} \mathrm{~s}^{-1}$ and a shell radius of $100 \mathrm{pc}$ after $3 \times 10^{6} \mathrm{yr}$, for the case of a uniform shell. Bruhweiler $e t$ al. assumed that the first supernova within such an association would not occur until after $3 \times 10^{6} \mathrm{yr}$. The age of Sco OB1 is estimated to be only $(3.6 \pm 0.6) \times 10^{6}$ yr (van Genderen, Bijeveld, and van Groningen 1984), and there appears to be no evidence that a supernova has yet occurred in the association. The two $\mathrm{H}$ II complexes in the vicinity, IC 4628 and RCW 113, are both thermal radio emitters (Wilson and Mezger 1970). The material seen expanding around the Sco OB1 stars therefore appears to be a good example of a shell caused by the effects of stellar mass loss, without the influence of supernova events.

\section{INTERPRETATION OF THE OBSERVED $\mathrm{Na} \mathrm{I/Ca} \mathrm{II} \mathrm{RATIOS}$}

It has long been known that interstellar absorption components can exhibit a very wide range of $\mathrm{Na}$ I/Ca II ratios, and this is now attributed chiefly to variations in the gas-phase calcium abundances. Jura (1975) and Phillips, Pettini and Gondhalekar (1984) have shown that, for sight lines with $0.03 \leq E(B-V) \leq 0.30$, the data are consistent with sodium having a constant gas-phase depletion factor of about a factor of 4. Jura (1976) argued that the widely different $\mathrm{Na}$ I/Ca II ratios observed along different lines of sight were due to variations in the gas-phase abundance of calcium, caused by depletion onto grains and grain destruction.

Routly and Spitzer (1952) first drew attention to the velocity dependence of the $\mathrm{Na}$ I/Ca II ratio, specifically that it tends to decrease with increasing absolute velocity. Siluk and Silk (1974) confirmed this result in an extensive survey of the then available data for 64 stars. Components with absolute velocities greater than $20 \mathrm{~km} \mathrm{~s}^{-1}$ were found to have much lower $\mathrm{Na}$ I/Ca II ratios than those typical of components with velocities less than this value. They found that "normal" components, those with $\left|v_{\text {LSR }}\right| \leq 20 \mathrm{~km} \mathrm{~s}^{-1}$, usually had Na I/Ca II ratios in the range $2-90$. This low-velocity material corre- sponds to the components lying between 0 and $-20 \mathrm{~km} \mathrm{~s}^{-1}$ in Figure 7. These have ratios in the range $1-200$, similar to those found for standard cool diffuse cloud components (e.g., Hobbs 1978b).

The lack of significant depletion of elements such as $\mathrm{Ca}, \mathrm{Fe}$, and $\mathrm{Si}$ in velocity components with $\left|v_{\mathrm{LSR}}\right|>70 \mathrm{~km} \mathrm{~s} \mathrm{~s}^{-1}$ (Jenkins, Silk, and Wallerstein 1976; Shull, York, and Hobbs 1977) was interpreted by Barlow and Silk (1977) as due to the destruction of refractory grains by sputtering in nonmagnetic shocks. Cowie (1978) and Shull (1978) showed that the gyromagnetic precessing of grains in magnetic shocks would enhance the efficiency of this process. Jura (1976) interpreted the velocity dependence of the observed $\mathrm{Ca}$ depletions in clouds as due to grain-grain collisions in shocks, while Shull (1977) investigated in more detail the enhancement of this mechanism by gyromagnetic precessing. Seab and Shull (1983) conclude that sputtering in shocks is effective in destroying grains of all sizes, while grain-grain collisions can enhance the destruction of the largest grains. However, the destruction of refractory grains by these various processes is ineffective at shock speeds of less than about $40 \mathrm{~km} \mathrm{~s}^{-1}$.

Barlow and Silk (1977) and Barlow (1978) have shown that the significant variations in gas-phase depletions that have been observed for metallic elements such as $\mathrm{Ca}, \mathrm{Ti}$, and $\mathrm{Fe}$ in components with velocities less than this can be explained by the desorption of adsorbed atoms from grain surfaces by lowvelocity shock sputtering. Considerable differential depletion of $\mathrm{Na}$ and $\mathrm{Ca}$ would be expected because of the much lower adsorption binding energy of $\mathrm{Na}$.

Phillips, Pettini and Gondhalekar (1984) showed that while the degree of depletion of $\mathrm{Na}$ was independent of the mean density along a sight line, that of $\mathrm{Ca}$ increased with increasing mean density. For a sample of nine metallic elements with depletion versus density data available, they found that the gradient of the relation between depletion factor and mean density was strongly correlated with $E_{\text {ads }} / A^{1 / 2}$, where $A$ is the atomic weight and $E_{\text {ads }}$ is the chemisorption binding energy onto the grain surface, taken from Barlow (1978). This supports the dynamic adsorption-desorption model for depletion in the low-velocity and intermediate-velocity components since the rate at which atoms stick to grain surfaces will be proportional to the gas density and to $A^{-1 / 2}$, while the time they remain on the surface, before being sputtered, should be proportional to $E_{\text {ads }}$.

Barlow and Silk (1977) and Barlow (1978) showed that in an intercloud medium with $n_{\mathrm{H}} \sim 0.2 \mathrm{~cm}^{-2}$ and $T \sim 5000 \mathrm{~K}$, the time scale for significant heavy element depletion would be longer than the mean interval between shocks, so that grain surfaces would be swept clean and the depletion factors would reflect those due to the underlying grains. In cool diffuse clouds the much higher densities ensure that significant depletion of elements such as $\mathrm{Ca}, \mathrm{Ti}$, and $\mathrm{Fe}$ would take place onto grains during the intervals between shocks. This accounts for Jenkins, Savage, and Spitzer's (1986) picture of the observed depletion along any given sight line as being due to a mixture of components having two different depletion states-the lowdepletion components correspond to warm low-density material whose grain surfaces are bare, while the highdepletion components correspond to cool diffuse interstellar clouds where a large fraction of the gas phase metals have been trapped on grain surfaces.

We interpret the velocity components in Figure 7 lying between 0 and $-20 \mathrm{~km} \mathrm{~s}^{-1}$ as arising from cool diffuse clouds in which calcium has undergone significant depletion onto 
grain surfaces, while the positive velocity components in Figure 7 are interpreted as arising from warm low-density material in which $\mathrm{Ca}$ only has its underlying depletion due to membership within refractory grains (Field 1974).

As discussed in $\S \mathrm{IV} f$, the high negative velocity components in Figure 7 probably originate within a swept-up shell around the Sco OB1 association, with an expansion velocity of 20-35 $\mathrm{km} \mathrm{s}^{-1}$ relative to the association. Shocks with velocities at the upper end of this range are capable of removing $\mathrm{Ca}$ from grain surfaces, thus producing lower $\mathrm{Na}$ I/Ca II ratios than for the low-velocity clouds. The fact that the $\mathrm{Na}$ I/Ca II ratios are not as low as for the positive velocity components may be because the relatively high densities characteristic of postshock regions lead to a high depletion rate that partially counteracts the desorption by shock sputtering.

The apparent dip in the $\mathrm{Na} \mathrm{I} / \mathrm{Ca}$ II ratios seen between -20 and $-40 \mathrm{~km} \mathrm{~s}^{-1}$ in Figure $7 \mathrm{can}$ be interpreted as due to blending of Sco OB1 shell components with intercloud-type components similar to those observed at positive velocities if, as discussed in $\S I V d$, components of this type can be found up to $\pm 20 \mathrm{~km} \mathrm{~s}^{-1}$ from the local systemic velocity anywhere along a line of sight. We have tested this idea by adding the mean column densities found for the high negative velocity $\left(v \leq-40 \mathrm{~km} \mathrm{~s}^{-1}\right.$ ) components of $\mathrm{Na} \mathrm{I}$ and $\mathrm{Ca}$ II (about $5 \times 10^{11}$ and $4 \times 10^{11} \mathrm{~cm}^{-2}$, respectively) to the mean column densities found for the positive velocity components $\left(\leq 0.8 \times 10^{11} \mathrm{~cm}^{-2}\right.$ for $\mathrm{Na}$ I and about $5 \times 10^{11} \mathrm{~cm}^{-2}$ for $\mathrm{Ca}$ II). Dividing the $\mathrm{Na}$ I total by the $\mathrm{Ca}$ II total gives a predicted value of $\log (\mathrm{Na} \mathrm{I} / \mathrm{Ca}$ II) of -0.2 , consistent with the dip observed in Figure 7 at velocities between -20 and $-30 \mathrm{~km}$ $\mathrm{s}^{-1}$.

Part of the Routly-Spitzer effect can be accounted for if the low $\mathrm{Ca}$-depletion intercloud components do indeed possess a wider range of velocities than the high $\mathrm{Ca}$-depletion cool clouds. At low velocities, the large column density of cool clouds would generally mask any intercloud components, so that the $\mathrm{Na}$ I/Ca II ratios characteristic of the latter would only be seen outside the velocity range occupied by interstellar clouds, accounting for the observed velocity dependence of the $\mathrm{Na}$ I/Ca II ratios for $0<\left|v_{\text {LSR }}\right|<40 \mathrm{~km} \mathrm{~s}^{-1}$ in Figure 1 of Siluk and Silk (1974). The low Na I/Ca II ratios of interstellar components with $\left|v_{\text {LSR }}\right|>40 \mathrm{~km} \mathrm{~s}^{-1}$ are, on the other hand, likely to be due to shocks and their associated dynamic grain destruction effects.

We thank the Director of the Mount Stromlo and Siding Spring Observatories for the generous allocation of telescope time. This work was carried out while I. A. C. was pursuing his Ph.D. studies at the University of London Observatory. The support and encouragement of Dr. Derek McNally during this period is warmly acknowledged by him. I. A. C. is also grateful for many stimulating conversations with Mr. P. C. T. Rees, also at ULO, particularly concerning the instrumental characteristics of the Mount Stromlo spectrograph. Mr. Rees is also gratefully acknowledged for making a number of useful modifications to the BACH program. We thank SERC for financial support, and the Centre de Données Stellaires, Strasbourg, for bibliographic information.
Avedisova, V. S. 1972, Soviet Astr. 15, 708.

Barlow, M. J. 1978, M.N.R.A.S., 183, 417 .

Barlow, M. J., and Silk, J. 1977, Ap. J. (Letters), 211, L83.

Bertout, C., Leitherer, C., Stahl, O., and Wolf, B. 1985, Astr. Ap., $144,87$.

Blades, J. C., Wynne-Jones, I., and Wayte, R. C. 1980, M.N.R.A.S., 193, 849.

Bok, B. J., Bok, P. F., and Graham, J. A. 1966, M.N.R.A.S., 131, 247.

Bruhweiler, F. C., Gull, T. R., Kafatos, M., and Sofia, S. 1980, Ap. J. (Letters), 238, L27.

Carrasco, L., and Crézé, M. 1978, Astr. Ap., 65, 279.

Cowie, L. L. 1978, Ap. J., 225, 887. Cowie, L. L., Hu, E. M., Taylor, W., and York, D. G. 1981, Ap. J. (Letters), 250,
L25.

Cowie, L. L., Songaila, A., and York, D. G. 1979, Ap. J., 230, 469.

Cowie, L. L., and York, D. G. 1978, Ap. J., 223, 876

Crawford, I. A., Rees, P. C. T., and Diego, F. 1987, Observatory, 107, 147.

Davenhall, C. 1977, University College London, internal memo.

Deutschman, W. A., Davis, R. J., and Schild, R. E. 1976, Ap. J. Suppl., 30, 97.

Dyson, J. E., and de Vries, J. 1972, Astr. Ap., 20, 223.

Ferlet, R., Vidal-Madjar, A., and Gray, C. 1985, Ap. J., 298, 838

Field, G. B. 1974, Ap. J., 187, 453.

Garrison, R. F., Hiltner, W. A., and Schild, R. E. 1977, Ap. J. Suppl., 35, 111.
Giacchetti, A., Stanley, R. W., and Zalubas, R. 1970, J.Opt. Soc. Am., 60, 474.

Herbst, W., and Havlen, R. J. 1977, Astr. Ap. Suppl., 30, 279.

Hobbs, L. M. 1969, Ap. J., 157, 135.

1974, Ap. J., 191, 381.

1978a, Ap.J., 222, 491.

1978b, Ap. J. Suppl., 38, 129
1984, Ap. J. Suppl., 56, 315

Hoffleit, D., and Jaschek, C. 1982, The Bright Star Catalogue (4th ed.; New

Haven: Yale University Observatory).

Howarth, I. D., and Phillips, A. P. 1986, M.N.R.A.S., 222, 809

Humphreys, R. M. 1978, Ap. J. Suppl., 38, 309.

Jenkins, E. B., Savage, B. D., and Spitzer, L. 1986, Ap. J., 301, 355

Jenkins, E. B., Silk, J., and Wallerstein, G. 1976, Ap. J. Suppl., 32, 681

Jura, M. 1975, Ap. J., 200, 415

. 1976, Ap. J., 206, 691. Keenan, F. P., Brown, P. J. F., Dufton, P. L., and Lennon, D. J. 1984, Ap. J.,
279, L11.

Kerr, F. J., and Lynden-Bell, D. 1986, M.N.R.A.S., 221, 1021.

Laurent, C., Paul, J. A., and Pettini, M. 1982, Ap. J., 260, 163.

\section{REFERENCES}

Laval, A. 1972, Astr. Ap., 21, 271.

Leitherer, C., and Chavarria-K, C. 1987, Astr. Ap., 175, 208.

Moore, C. E., Minnaert, M. G. J., and Houtgast, J. 1966, The Solar Spectrum $2935 A$ to 8770 A (NBS Monograph 61). (Washington, D.C.: US Department of Commerce).

Morton, D. C., and Smith, W. H. 1973, Ap. J. Suppl., 26, 333.

Munch, G. 1957, Ap. J., 125, 42.

Norlén, G. 1973, Phys. Scripta, $8,249$.

Pettini, M., and Gillingham, P. 1987, AAO Newsletter, 42, 4

Phillips, A. P., and Gondhalekar, P. M. 1981, M.N.R.A.S., 196, 533.

Phillips, A. P., Pettini, M., and Gondhalekar, P. M. 1984, M.N.R.A.S., 206, 337.

Phillips, A. P., Welsh, B. Y., and Pettini, M. 1984, M.N.R.A.S., 206, 55.

Pikelner, S. B. 1968, Ap. Letters, 2, 97.

Routly, P. M., and Spitzer, L. 1952, Ap. J., 115, 227.

Savage, B. D., Bohlin, R. C., Drake, J. F., and Budich, W. 1977, Ap. J., 216, 291.

Schild, R. E., Neugebauer, G., and Westphal, J. A. 1971, A.J., 76, 237 .,

Schild, R. E., Garrison, R. F., and Hiltner, W. A. 1983, Ap. J. Suppl., 51, 321.

Seab, C. G., and Shull, J. M. 1983, Ap. J., 275, 652.

Shull, J. M. 1977, Ap. J., 215, 805.

Shull. 1978, Ap. J., 226, 858.

Shull, J. M., York, D. G., and Hobbs, L. M. 1977, Ap. J. (Letters), 211, L139.

Siluk, R. S., and Silk, J. 1974, Ap. J., 192, 51.

Spitzer, L. 1978, Physical Processes in the Interstellar Medium (New York: John Wiley)

Stapinski, T. E., Rodgers, A. W., and Ellis, M. J. 1979, Adv. Electronics Electron Phys., 52, 389.

Phy 1981, Pub. A.S.P., 93, 242.

Strömgren, B. 1948, Ap. J., 108, 242.

Struve, O. 1944, Ap.J., 100, 189 .

van Genderen, A. M., Bijeveld, W., and van Groningen, E. 1984, Astr. Ap. Suppl., 58, 537 .

Vidal-Madjar, A., Ferlet, R., Gry, C., and Lallement, R. 1986, Astr. Ap., 155,

Walborn, N. R., and Hesser, J. E. 1975, Ap. J., 199, 535.

.1982, Ap. J., 252, 156.

Wayte, R. C., Wynne-Jones, I., and Blades, J. C. 1978, M.N.R.A.S., 182, 5P.

Weaver, R., McCray, R., Castor, J. J., Shapiro, P., and Moore, R. 1977, Ap. J., 218, 377.

Whiteoak, J. B., and Gardner, F. F. 1985, Proc. Astr. Soc. Australia, 6, 164.

Wilson, T. L., and Mezger, P. G. 1970, Ap. Letters, 5, 47.

M. J. BARLOW: Department of Physics and Astronomy, University College London, Gower Street, London WC1E 6BT, UK

J. C. Blades: Space Telescope Science Institute, 3700 San Martin Drive, Baltimore, MD 21218

I. A. Crawford: Department of Physics and Astronomy, University College London, Gower Street, London WC1E 6BT, UK 